\title{
Standardized motor assessments before the age of five predicting school-aged motor outcome including DCD: A systematic review
}

\author{
Amy De Roubaix ${ }^{\mathrm{a},{ }^{*} \text {, Dominique Van de Velde }}{ }^{\mathrm{a}}$, Herbert Roeyers ${ }^{\mathrm{b}}$, Hilde Van Waelvelde ${ }^{\mathrm{a}}$ \\ a Faculty of Medicine and Health Care Sciences, Department of Rehabilitation Sciences, Ghent University, Entrance 46, UZ Ghent, C. Heymanslaan 10, 9000, \\ Ghent, Belgium \\ ${ }^{\mathrm{b}}$ Faculty of Psychology and Educational Sciences, Department of Experimental Clinical and Health Psychology, Ghent University, Henri Dunantlaan 2, 9000, \\ Ghent, Belgium
}

\section{A R T I C L E I N F O}

\section{Article history:}

Received 16 July 2020

Received in revised form

10 November 2020

Accepted 5 December 2020

\section{Keywords:}

Developmental coordination disorder

Child

Prediction

Motor development

Sensitivity and specificity

complex Minor Neurological Disorder

\begin{abstract}
A B S T R A C T
Aim: Developmental Coordination Disorder (DCD) is a common neurodevelopmental disorder usually diagnosed at primary-school-age. This systematic review aimed to summarize available standardized motor assessments before five years of age predicting DCD, complex Minor Neurological Disorder (cMND) and motor delay assessed by a standardized motor test.

Methods: A systematic search was performed in MEDLINE, CINAHL, WoS, Scopus, CENTRAL and ERIC. A hand search was executed. Only data of non-Cerebral Palsy children was included.

Results: At or before two years, the BSID, motor subtests of GMDS, NOMAS, and NSMDA might be valuable in detecting school-aged motor delay, while starting at three years, the PDMS, motor subtests of GMDS, NSDMA, M-ABC-2, and CAMPB show promising results. General movements Assessment is associated with CMND, but does not seem sensitive enough to detect DCD. Predictive values are superior in high-risk groups and improve as children age. However, no assessment instrument reached $80 \%$ sensitivity and specificity.

Conclusion: Standardized motor assessments before five years seem valuable in detecting early motor problems. More longitudinal research commencing in infancy, including multiple assessments over time and the implementation of clear diagnostic criteria is imperative.
\end{abstract}

(C) 2020 European Paediatric Neurology Society. Published by Elsevier Ltd. All rights reserved.

\section{Contents}

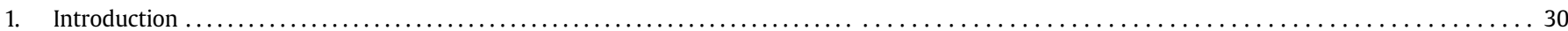

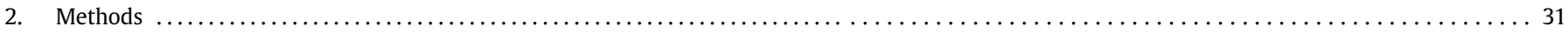

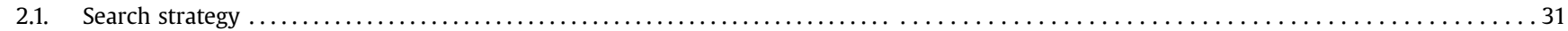

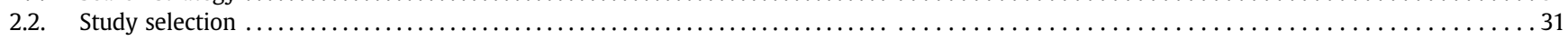

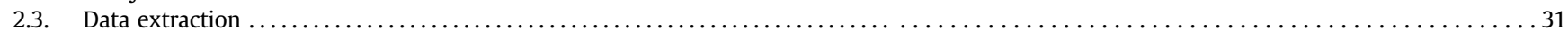

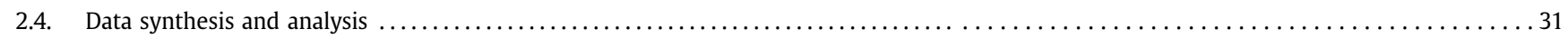

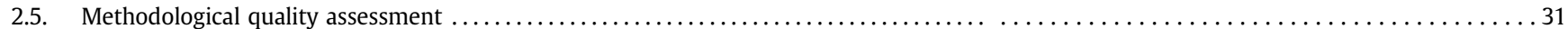

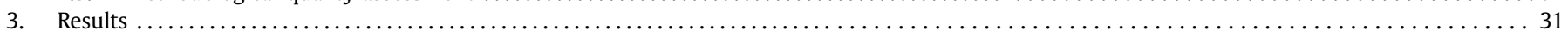

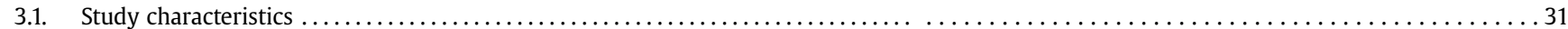

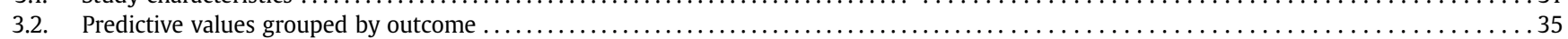

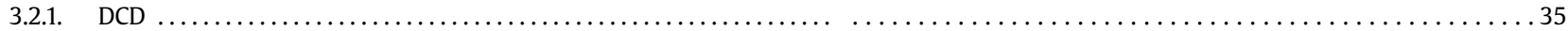

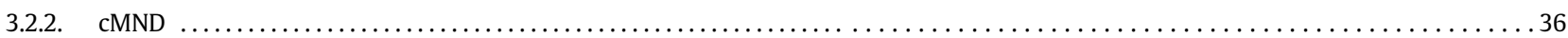

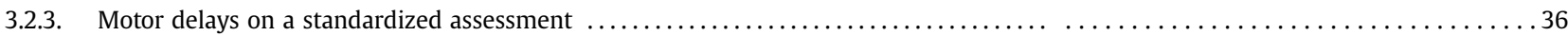

\footnotetext{
* Corresponding author. Department of Rehabilitation Sciences, Ghent University, Entrance 46, UZ Ghent, C. Heymanslaan 10, 9000, Ghent, Belgium.

E-mail addresses: amy.deroubaix@ugent.be (A. De Roubaix), Dominique. vandevelde@ugent.be (D. Van de Velde), Herbert.roeyers@ugent.be (H. Roeyers), Hilde.vanwaelvelde@ugent.be (H. Van Waelvelde).
} 


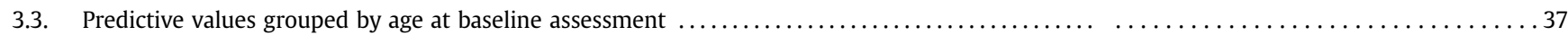

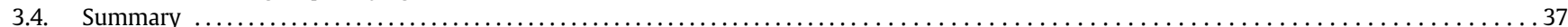

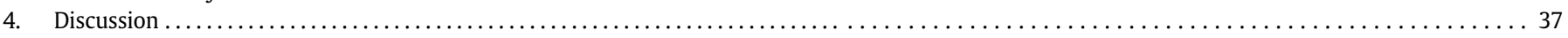

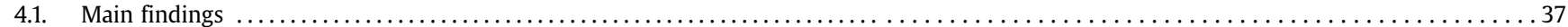

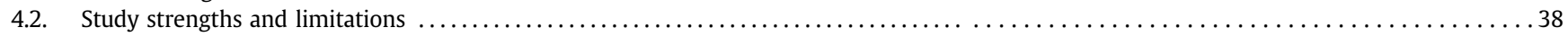

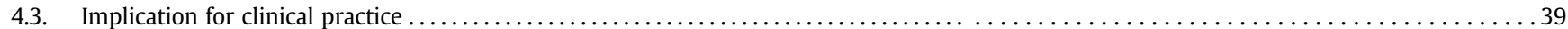

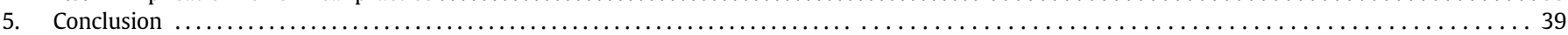

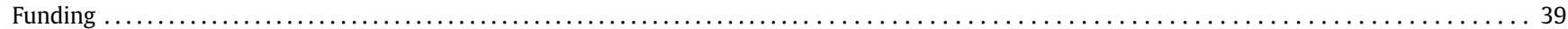

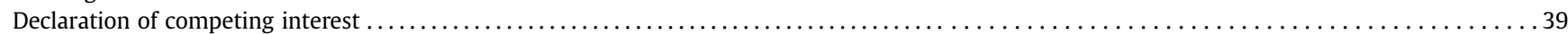

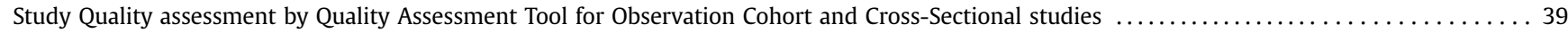

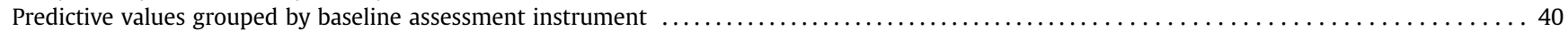

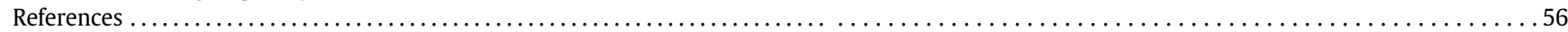

\begin{tabular}{|c|c|c|}
\hline Nomenclature & $\begin{array}{l}\text { GMDS } \\
\text { M }\end{array}$ & $\begin{array}{l}\text { Griffiths Mental Development Scales } \\
\text { Month(s) }\end{array}$ \\
\hline Albert Infant Motor Scale & \multirow{2}{*}{\multicolumn{2}{|c|}{$\begin{array}{l}\text { M-ABC(-NL) Movement Assessment Battery for Children - } \\
\text { (Dutch Version) }\end{array}$}} \\
\hline Ages and Stages Questionnaire & & \\
\hline \multirow{2}{*}{$\begin{aligned} & \text { BOT-(2)-(MP) (SF) } \text { Bruininks-Oseretsky Test - (Second Edition) - } \\
& \text { (of Motor Proficiency) (Short Form) }\end{aligned}$} & MOS & Motor Optimality Score \\
\hline & MPU & Motor-Perceptual Development \\
\hline Combined Assessment of Motor Performance and & NOMAS & Neonatal Oral Motor Assessment Scale \\
\hline Behavior & NSMDA & Neuro-Sensory Motor Developmental Assessment \\
\hline (complex) Minor Neurological Dysfunction & PDI & Psychomotor Developmental Index \\
\hline Cerebral Palsy & PMA & Post-menstrual age \\
\hline $\operatorname{Day}(s)$ & PDMS & Peabody Developmental Motor Scales \\
\hline
\end{tabular}

\section{Introduction}

The Diagnostic and Statistical Manual fifth edition (DSM-V) defines Developmental Coordination Disorder (DCD) as early-onset (criterion $C$ ) deficits in acquiring and executing motor coordination skills (criterion A) [1]. These deficits significantly interfere with the performance of activities of daily living and impact on academic productivity, leisure, and play (criterion B). Difficulties cannot be attributed to other conditions, such as intellectual disability, visual impairment, or other neurological disorders that affect movement (criterion D). As advised by the European Academy of Childhood Disability (EACD), the diagnosis of DCD preferably occurs at primary-school-age [2]. Nevertheless, a growing body of literature recognizes the importance of early detection $[3,4]$ as DCD may have a vast impact on the quality of life [5] and is associated with important secondary problems. The lower motor competence of these children has been associated with less vigorous physical activity, higher body fatness, lower physical fitness, and lower healthrelated quality of life [6-8]. Additionally, higher levels of motor impairment have been associated with less friendships and lower peer acceptance [9]. Unsurprisingly, the socio-emotional impact is high as these children often develop low self-esteem and experience significantly more emotional and behavioral problems $[7,10]$. Furthermore, children with DCD participate less in home, school, and community settings [11]. Having a diagnosis of DCD greatly impacts the emotional, social, and financial well-being of the entire family [12]. Nevertheless, DCD and related terms such as dyspraxia are among the least known childhood disorders and too often remain unrecognized [13]. Many children with autism spectrum disorders, attention deficit hyperactivity disorder, and learning disorders likewise experience motor problems, sometimes resulting in a co-morbid diagnosis of DCD. Yet, motor problems are frequently considered a feature of another disorder or are simply overlooked. Minor Neurological Disorder (MND) is a condition detected by a standardized neurological examination which is strongly related to DCD $[14,15]$. The criteria for MND are agespecific and based on the number of dysfunctional neurological domains such as posture and tone, coordination and fine manipulative ability. While the first type 'simple MND' has limited clinical relevance and indicates that children have a typical but nonoptimal brain function presenting a deviation in only a limited number of domains, the second type 'complex MND' (cMND) is clinically relevant as it is seen in children with non-optimal brain functions in several domains. Approximately 59\% of children with probable DCD also adhere to the criteria for cMND [16]. Early detection and referral of DCD may considerably improve the health, socio-emotional, and educational outcomes of children with DCD $[17,18]$. However, accurate early detection is challenging because of the heterogeneous character of DCD and the great variability in early child development. A standard pediatric assessment is often 
not sensitive enough to detect motor difficulties, therefore a standardized motor assessment is recommended when evaluating motor development in young children [19].

Several systematic reviews described the psychometric properties and predictive values of a great variety of early motor assessments. However, all reviews included a heterogeneous group of children with mixed mild and more severe neurological disorders such as Cerebral Palsy (CP) [20-22]. Additionally, most reviews focused only on very young children ( $<2$ years). To the best of our knowledge, no review has focused primarily on the predictive values of early motor assessments regarding DCD, CMND, and significant motor delay on a standardized test at school-age. Therefore, this review aims to answer the following research question: Which standardized motor assessments before the age of five are available to accurately predict DCD, cMND, and significant motor delay on a standardized assessment in children aged five to twelve years(y)?

\section{Methods}

This review follows the Preferred Reporting Items for Systematic Reviews and Meta-Analysis (PRISMA) statement [23]. The review protocol has been registered and can be accessed on PROSPERO (https://www.crd.york.ac.uk/prospero/; registration number: CRD42018105599).

\subsection{Search strategy}

Exhaustive search strategies were developed and conducted by the first author of this article (ADR). Seven databases (MEDLINE (PubMed), Embase (Embase.com), CINAHL (EBSCOhost), Web of Science, Scopus, CENTRAL, and ERIC) were searched on 06/08/2018 and updated on $11 / 06 / 2020$. The full search strategies can be consulted on PROSPERO.

\subsection{Study selection}

Studies were screened by two authors independently and were included if the following criteria were met: (1) observational studydesign with follow-up period $>1$ year; (2) two standardized motor assessments, one performed before mean age of $5 \mathrm{y}$ and one between the mean age of 5y and 12y; (3) written in English, Dutch or French; (4) peer-reviewed published full-text articles. The chosen age-categories reflect the EACD-guidelines [24] advising to diagnose children with DCD preferably after 5y. The maximum age was set at $12 \mathrm{y}$, excluding adolescents and adults whose diagnostic processes may be different. Studies were excluded for the following reasons: (1) interventional studies; (2) total sample size <20; (3) no report of a separate motor score; (4) studies focusing on neurological or cognitive assessments were excluded, unless a specific motor subtest was reported. When studies comprised children with $\mathrm{CP}$, studies were only witheld when it was possible to deduct motor scores of non-CP-children or when authors provided the specific data on request. If not published, authors were asked to provide additional information on cross-tabs values. Multiple articles of the same study were included if different motor outcomes were presented in each publication.

\subsection{Data extraction}

Data extraction was performed independently by the first author using the following headings: author(s), number of participants, participant characteristics, name assessment, mean age at assessment, applied cut-off scores and the predictive values (specificity, sensitivity, positive predictive value (PPV), negative predictive value (NPV), Area Under the Curve (AUC), correlation and regression coefficients, $\mathrm{r}^{2}$, Odds Ratio (OR) and Likelihood Ratio (LR)) with significance levels. The authors of eight articles supplied additional data. Contingency tables $(2 \times 2)$ were extracted or constructed with the provided dichotomized data (presence/ absence of a motor problem at baseline versus presence/absence of a motor problem at outcome assessment). To enhance comparison between studies applying General movements Assessment (GmA), only data reporting the quality of general movements or items of the Motor Optimality Score (MOS) are discussed in this review. Hence, analysis of specific GmA characteristics on item-level (e.g. presence of a specific movement pattern), the quantity of general movements, combinations of different items, or any other criteria related to general movements were not discussed.

\subsection{Data synthesis and analysis}

The myriad of outcome measures made it difficult to group results and prohibited a meta-analysis. Therefore, the results are discussed through a narrative summary per assessment instrument and outcome category. To enhance comparison, the following missing predictive values were calculated using MedCalc: sensitivity, specificity, PPV, NPV, relative risk (RR) and associated 95\% Confidence Intervals (CI). Sensitivity refers to the percentage of children with a motor problem at school-age who also had a poor motor score before $5 y$ of age. Specificity refers to the percentage of children without a motor problem at school-age who also had a good motor score before the age of $5 \mathrm{y}$. Positive predictive value is the probability that the child will have motor problems at schoolage if they have a poor motor score before $5 \mathrm{y}$ of age. Negative predictive value is the probability that the child will have a good motor performance at school-age if they have a good motor score before the age of $5 \mathrm{y}$. If not reported and the necessary data was available, $\mathrm{Chi}^{2}$ or Fisher-exact tests were conducted to determine the probability that the two points of measurement were coincidently related.

\subsection{Methodological quality assessment}

The methodological quality of each article was assessed by two authors independently using the Quality Assessment Tool for observational cohort and cross-sectional studies [25]. Study quality was rated as good (score $\geq 10$ ), fair $(7-9)$, or poor $(\leq 6)$ based on 14 items.

\section{Results}

The database search resulted in 3729 articles whereof 3559 articles were withheld after deduplication. Two authors (ADR and HVW) independently performed the first screening, resulting in a remainder of 78 articles. Disagreements were resolved by consensus. After the second screening 23 articles were withheld. Almost perfect overall agreement was established at 92\% (Cohen's kappa $\kappa=0.8$ ). A hand search was completed, applying both backward $(n=6)$ and forward reference tracking $(n=4)$ on the included articles. This resulted in a total number of 33 included articles regarding 31 distinct studies. A detailed flow-chart of the selection process can be found in Fig. 1.

\subsection{Study characteristics}

The participant and study characteristics of the included studies are summarized in Table 1. Substantial clinical and methodological heterogeneity was present. The majority of studies were published after 2000. Most studies comprised high-risk children born either 


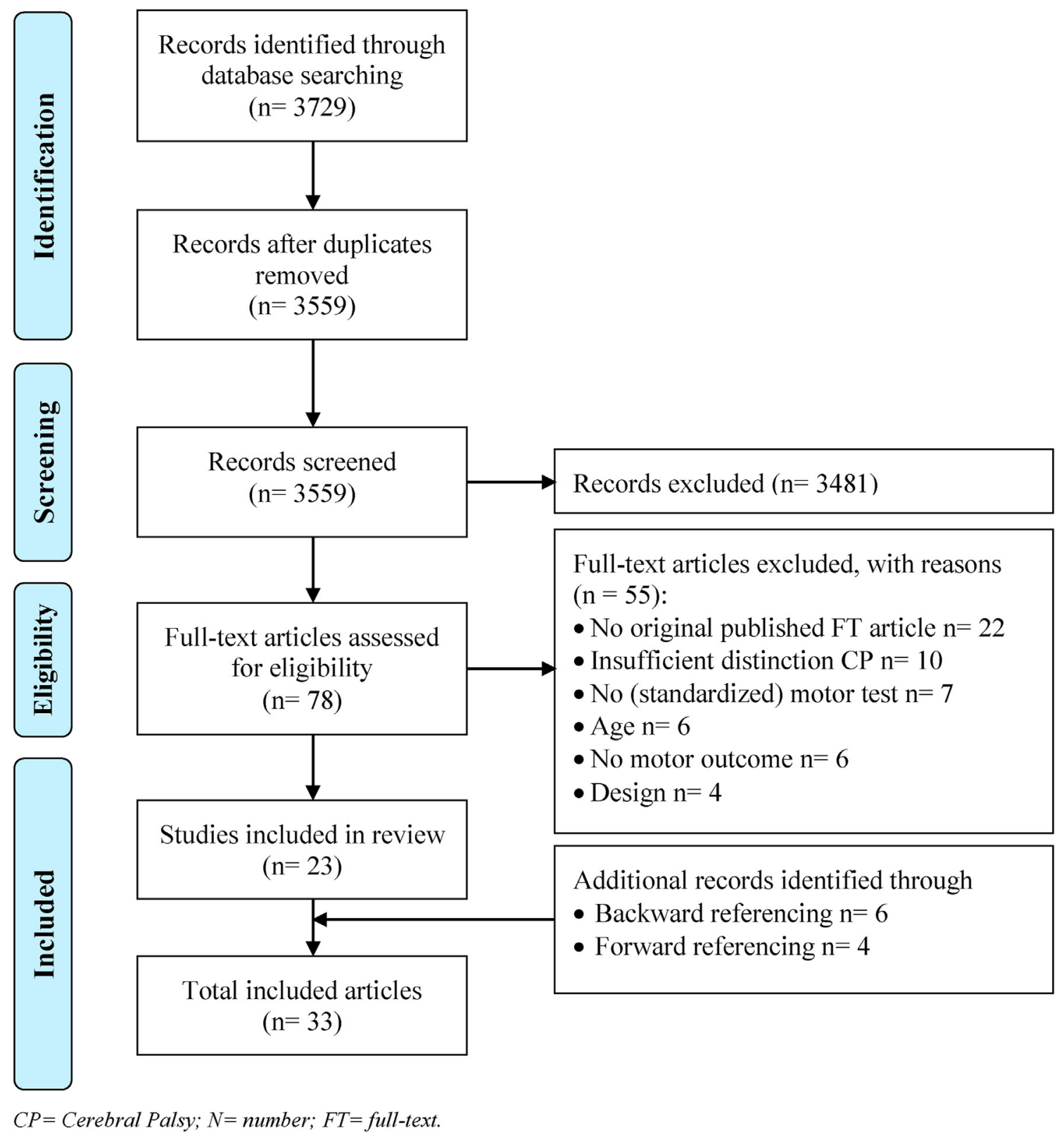

Fig. 1. PRISMA flow diagram summarising the search process.

preterm $(\mathrm{n}=15)$ or with severe neonatal complications or congenital anomalies $(\mathrm{n}=3)$, whereas six studies comprised term low-risk children and another seven studies covered mixed groups of high- and low-risk infants.

A total of fourteen distinctive instruments were applied before 5y: GmA[26-39], Bayley Scales of Infant Development (BSID) [36,40-48], MOS, Peabody Developmental Motor Scales (PDMS) [49-51], Alberta Infant Motor Scale (AIMS) [47,48], Motor subtests of the Griffiths Mental Development Scales (GMDS) [50,52], Ages and Stages Questionnaire (ASQ) [53,54], Movement Assessment Battery for Children (M-ABC) [55], Neurological, Sensory, Motor, Developmental Assessment (NSMDA) [56], Motor scale of the Neonatal Behavioral Assessment Scale (NBAS), Combined Assessment of Motor Performance and Behavior (CAMPB) [57], Neonatal Oral-Motor Assessment Scale (NOMAS) [58] and NEPSY Copy Design Task [54]. Seventeen studies reported motor assessments before 6 months (m) [26-35,37-40,43,44,48,53,58], fifteen between $6 \mathrm{~m}$ and $2 \mathrm{y}[36,40-44,46-53,56]$ and eight between 2 and $5 y$ [45,49,50,53-57]. Within the baseline assessment age of this review (before 5y), eight studies evaluated children twice
[26,30,32,33,36,38,41,50,52], seven studies evaluated children three times [27,28,30,40,42,46,47,56], and six studies evaluated children four times or more [36,43,44,48,51,58]. Outcome measures were defined as (probable) DCD in four studies, cMND in five studies and significant motor delay on a standardized assessment in 23 studies. Follow-up length varied greatly between two and eleven years.

Predictive values (Sensitivity, specificity, PPV, NPV, or RR) were available in seventeen studies. Correlation coefficients were available in nine studies while another fifteen studies accounted for confounding variables applying various statistical models. No expedient data could be extracted out of three articles [34,49,51] leaving 30 articles with fitting data.

Study quality was rated as good in 20 articles $[27,28,30,32-34,36-38,40,42,45-49,52,55,56,58]$ fair in eleven articles [26,29,31,35,39,41,50,51,53,54,57] and poor in two articles $[43,44]$ (Appendix A). Total scores ranged between 4.5 and 12.5. Item five (power analyses, effect size calculation) was most frequently scored absent. Next, many studies did not provide sufficient details on the psychometric properties of the used 
Table 1

Participant and study characteristics of the included studies.

\begin{tabular}{|c|c|c|c|c|c|}
\hline Author(s) & Type participants & Baseline assessment & $\begin{array}{l}\text { Mean age at } \\
\text { baseline } \pm \mathrm{SD} \\
\text { (range) }\end{array}$ & Outcome assessment & $\begin{array}{l}\text { Mean age at outcome } \pm \text { SD } \\
\text { (range) }\end{array}$ \\
\hline $\begin{array}{l}\text { Barnett et al., } \\
2003 \text { [52] }\end{array}$ & $\begin{array}{l}\text { FT infants with neonatal } \\
\text { encephalopathy }\end{array}$ & $\begin{array}{l}\text { GMDS <-1SD subtests (1) } \\
\text { Locomotor Scale, (2) Eye-hand } \\
\text { Scale }\end{array}$ & $\begin{array}{l}12 \mathrm{~m}(9 \mathrm{~m} \\
-15 \mathrm{~m}), 24 \mathrm{~m} \\
(21 \mathrm{~m}-27 \mathrm{~m})\end{array}$ & M-ABC-I Total Score Pc $<15$ & $5.5 y-6.5 y$ \\
\hline $\begin{array}{l}\text { Bruggink } \\
\text { et al., 2008, } \\
2009 \\
{[27,28]}\end{array}$ & PT infants $(G A<32 w)$ & $\begin{array}{l}\text { GmA by Prechtl: quality of GMs } \\
\text { MOS }\end{array}$ & $\begin{array}{l}6-10 w, 11- \\
16 w, 17-21 w\end{array}$ & $\begin{array}{l}\text { cMND by Examination of the child with } \\
\text { MND (1979) and subcategories: (1) } \\
\text { Posture and muscle tone, (2) Reflexes, } \\
\text { (3) Choreiform dyskinesia, ( } 4 \text { ) } \\
\text { Coordination and balance, (5) Fine } \\
\text { manipulative ability, (6) Rare } \\
\text { dysfunctions (incl. excessive associated } \\
\text { movements). }\end{array}$ & $7-11 y$ \\
\hline $\begin{array}{l}\text { Danks et al., } \\
2012[56]\end{array}$ & $\begin{array}{l}\text { PT non-disabled ELBW } \\
(\mathrm{BW}<1000 \mathrm{~g})\end{array}$ & $\begin{array}{l}\text { NSMDA Total score and subtests: } \\
\text { (1) Gross motor skills, (2) Fine } \\
\text { motor skills, (3) Neurological } \\
\text { status, (4) Postural reactions, (5) } \\
\text { Sensory motor function }\end{array}$ & $8 \mathrm{~m}, 2 \mathrm{y}, 4 \mathrm{y}$ & M-ABC-I Total score Pc $<5$ & $\begin{array}{l}\text { Mean } 12.4 \mathrm{~m} \pm 0.70 \mathrm{~m}(11- \\
13 \mathrm{y})\end{array}$ \\
\hline $\begin{array}{r}\text { Eldred et al., } \\
2010 \text { [59] }\end{array}$ & $\begin{array}{l}\text { Convenience sample of infants } \\
\text { without developmental concerns }\end{array}$ & $\begin{array}{l}\text { PDMS-I and PDMS-2 Pc } \leq 16 \\
\text { subtests (1) Fine Motor Scale, (2) } \\
\text { Gross Motor Scale }\end{array}$ & $\begin{array}{l}9 \mathrm{~m}, 11 \mathrm{~m} \\
13 \mathrm{~m}, 16 \mathrm{~m} \\
21 \mathrm{~m}\end{array}$ & PDMS-2 Pc $\leq 16$ Gross Motor Scale & $5 y, 5.5 y$ \\
\hline $\begin{array}{c}\text { Evensen et al., } \\
2009 \text { [41] }\end{array}$ & $\begin{array}{l}\text { Mixed group of prematurely born } \\
\text { children with VLBW, FT children } \\
\text { with SGA and FT controls with } \\
\text { normal BW. }\end{array}$ & BSID-I PDI $<2$ SD & $1 \mathrm{y}$ & $\begin{array}{l}\text { PDMS-I Pc } \leq 5 \text { on } \geq 1 \text { subtest (norms } \\
\text { calculated by control group) }\end{array}$ & $5 y$ \\
\hline $\begin{array}{l}\text { Fjørtoft et al., } \\
2015 \text { [29] }\end{array}$ & VLBW infants $(<1500 g)$ & $\begin{array}{l}\text { GmA by Prechtl: quality of GMs } \\
\text { MOS }\end{array}$ & $14 w$ & M-ABC-2 Total Score Pc $\leq 16$ & $10-11 y$ \\
\hline $\begin{array}{l}\text { Goyen et al., } \\
2008 \text { [50] }\end{array}$ & $\begin{array}{l}\text { Apparently normal high-risk } \\
\text { children attending normal school } \\
\text { (PT NICU infants) and controls } \\
\text { (unclear if controls were included } \\
\text { in predictive analysis) }\end{array}$ & $\begin{array}{l}\text { GMDS Pc }<43 \text { on subscales ( } 1 \text { ) } \\
\text { Locomotor scale, ( } 2 \text { ) Eye-Hand } \\
\text { scale } \\
\text { PDMS-I subscales ( } 1 \text { ) Pc }<27 \text { Fine } \\
\text { motor, (2) Pc }<41 \text { Gross motor }\end{array}$ & $\begin{array}{l}1 \mathrm{y}, 3 \mathrm{y} \\
3 \mathrm{y}\end{array}$ & $\begin{array}{l}\text { Indication of DCD defined as M-ABC-I } \\
\mathrm{PC}<15\end{array}$ & $\begin{array}{l}\text { High-risk group: mean } \\
8,8 y \pm 0.3 ; \text { Controls: mean } \\
8,8 y \pm 0,4\end{array}$ \\
\hline $\begin{array}{r}\text { Goyen et al., } \\
2002 \text { [49] }\end{array}$ & $\begin{array}{l}\text { Apparently normal high-risk } \\
\text { infants }(\mathrm{GA}<29 \mathrm{w} \text { or } \mathrm{BW}<1000 \mathrm{~g}) \\
\text { attending normal schools }\end{array}$ & $\begin{array}{l}\text { PDMS-I mild-significant deficit } \\
\text { (undefined) at subtest (1) Gross } \\
\text { Motor Scale, (2) Fine Motor Scale }\end{array}$ & $18 \mathrm{~m} \mathrm{CA}, 3 \mathrm{y}$ & $\begin{array}{l}\text { PDMS-I subtests ( } 1 \text { ) Gross Motor Scale, } \\
\text { (2) Fine Motor Scale }\end{array}$ & $5 y$ \\
\hline $\begin{array}{l}\text { Griffiths et al., } \\
2017 \text { [55] }\end{array}$ & VPT infants $(G A<30 w)$ & $\begin{array}{l}\mathrm{M}-\mathrm{ABC}-2 \text { Total score } \mathrm{Pc} \leq 5 \text { and } \\
\mathrm{Pc} \leq 15\end{array}$ & $\begin{array}{l}4 y 4 m(4 y- \\
5 y 5 m)\end{array}$ & M-ABC-2 Total Score Pc $\leq 5$ and $\mathrm{Pc} \leq 15$ & $7 y 11 m(7 y-9 y 9 m)$ \\
\hline $\begin{array}{l}\text { Groen et al., } \\
2005[30]\end{array}$ & $\begin{array}{l}\text { High-risk (PT-birth associated } \\
\text { problems or FT with HIE and low- } \\
\text { risk infants }\end{array}$ & $\begin{array}{l}\text { GmA by Hadders-Algra: Quality of } \\
\text { GMs }\end{array}$ & $\begin{array}{l}<38 w \text { PMA, } \\
38 w-7 w \\
\text { PMA, } 8-17 w \\
\text { PMA }\end{array}$ & $\begin{array}{l}\text { cMND by 'Examination of the child with } \\
\text { Minor Neurological dysfunction'. }\end{array}$ & $\begin{array}{l}\text { Controls: mean } 137 \mathrm{~m}(113 \\
-150) \text {; Term high-risk: } \\
\text { mean } 111 \mathrm{~m} \text { (107-118); PT } \\
\text { high-risk: mean } 115 \mathrm{~m} \\
(108-126)\end{array}$ \\
\hline $\begin{array}{l}\text { Grunewald } \\
\text { et al., } 2014 \\
\text { [31] }\end{array}$ & ELBW children $(B W<1000 g)$ & $\begin{array}{l}\text { GmA by Prechtl: Quality of GMs } \\
\text { MOS }\end{array}$ & $14 \mathrm{w}( \pm 1,6 \mathrm{w})$ & $\begin{array}{l}\text { M-ABC-2 } \\
\text { Beery-VMI-4 }\end{array}$ & $10 \mathrm{y} 2 \mathrm{~m} \pm 0,8 \mathrm{~m}$ \\
\hline $\begin{array}{l}\text { Hadders- } \\
\quad \text { Algra et al., } \\
\text { 1999, 2004 } \\
{[32,33]}\end{array}$ & $\begin{array}{l}\text { High-risk (FT with HIE or PT with } \\
\text { neonatal complications) and low- } \\
\text { risk children (FT without } \\
\text { complications) }\end{array}$ & $\begin{array}{l}\text { GmA by Hadders-Algra: Quality of } \\
\text { GMs } \\
\text { MOS }\end{array}$ & $\begin{array}{l}36 w \text { PMA - } \\
8 w \text { post- } \\
\text { term, } 8-17 w \\
\text { post-term }\end{array}$ & $\begin{array}{l}\text { cMND by 'Neurological Examination of } \\
\text { the Child with Minor Neurological } \\
\text { Dysfunction' }\end{array}$ & Median 5\%o(4-9y) \\
\hline $\begin{array}{l}\text { Hamer et al., } \\
2016[34]\end{array}$ & $\begin{array}{l}\text { Children with definitely abnormal } \\
\text { Gms in infancy }\end{array}$ & $\begin{array}{l}\text { GmA by Hadders-Algra: frequency } \\
\text { of GMs }\end{array}$ & $\begin{array}{l}\text { Median 10w } \\
\text { CA }(9-13 w)\end{array}$ & $\begin{array}{l}\text { DCD-Q } \\
\text { VABS Total Index Score }<85\end{array}$ & $\begin{array}{l}\text { Median 8y4m (7y6m - } \\
10 \mathrm{y} 1 \mathrm{~m})\end{array}$ \\
\hline $\begin{array}{l}\text { Hemgren } \\
\text { et al., } 2008 \\
\text { [57] }\end{array}$ & $\begin{array}{l}\text { NICU children (PT and FT) without } \\
\text { major impairments }\end{array}$ & CAMPB & $\begin{array}{l}3 y \mathrm{CA} \\
( \pm 1 \mathrm{mo})\end{array}$ & $\begin{array}{l}\text { Moderate DCD (Pc }<15 \text { TOMI + Floor } \\
\text { level MPU }<4 y+\text { No general medical } \\
\text { condition, PDD or mental retardation } \\
\text { with floor level MPU }<3 y) \\
\text { Definite DCD (Pc }<5 \text { TOMI + Floor level } \\
\text { MPU }<4 y+\text { No general medical } \\
\text { condition, PDD or mental retardation } \\
\text { with floor level MPU }<3 y)\end{array}$ & $6.5 \mathrm{y} \mathrm{CA} \pm 1 \mathrm{~m}$ \\
\hline $\begin{array}{l}\text { Hitzert et al., } \\
2014 \text { [35] }\end{array}$ & $\begin{array}{l}\text { FT healthy children (Overlapping } \\
\text { cohort with Roze et al., 2010) }\end{array}$ & $\begin{array}{l}\text { GmA by Prechtl: Quality of GMs } \\
\text { MOS }\end{array}$ & $\begin{array}{l}\text { Median } 12.9 \\
\text { w }(9,3 \\
-18,6 w)\end{array}$ & $\begin{array}{l}\text { M-ABC-I-NL Total Score Pc }<15 \text { and } \\
\text { Pc }<5 \text { NEPSY-II Copy Design Task } \\
\text { Pc }<15 \\
\text { DCD-Q }\end{array}$ & $\begin{array}{l}\text { Median 5y } 11 \mathrm{~m}(5 \mathrm{y} 8 \mathrm{~m}- \\
7 \mathrm{y} 6 \mathrm{~m})\end{array}$ \\
\hline $\begin{array}{l}\text { Howe et al., } \\
2016 \text { [47] }\end{array}$ & $\begin{array}{l}\text { PT LBW children }(G A \leq 32 w ; \\
\text { BW } \leq 1500 \mathrm{~g})\end{array}$ & $\begin{array}{l}\text { AIMS } \\
\text { BSID-II PDI }\end{array}$ & $\begin{array}{l}12 \mathrm{~m} \mathrm{CA} \text { and } \\
18 \mathrm{~m} \mathrm{CA} \\
12 \mathrm{~m}, 18 \mathrm{~m} \\
\text { and } 24 \mathrm{~m}\end{array}$ & $\begin{array}{l}\text { M-ABC-I Total Score Pc } 5-15 \text { and Pc }<5 \\
\text { VABS - Chinese version: subtest motor } \\
\text { adaptive skills }\end{array}$ & $60.21 \mathrm{mo} \pm 1.87 \mathrm{mo}$ \\
\hline $\begin{array}{l}\text { Janssen et al., } \\
2016 \text { [46] }\end{array}$ & $\begin{array}{l}\text { VPT children }(G A<32 w) \text { without } \\
\text { severe impairment admitted to } \\
\text { NICU }\end{array}$ & $\begin{array}{l}\text { BSID-II (American Version) PDI } \leq 69 \\
\text { and PDI } 70-84\end{array}$ & $\begin{array}{l}6 \mathrm{~m} 1 \mathrm{w} \\
( \pm 2 \mathrm{w}) \\
12 \mathrm{~m} 0 \mathrm{w} \\
( \pm 2 \mathrm{w}) \\
24 \mathrm{~m} 3 \mathrm{w} \\
( \pm 3 \mathrm{w})\end{array}$ & M-ABC-2-NL Pc $<15$ & $63 m 1 w \pm 4 w$ \\
\hline
\end{tabular}


Table 1 (continued)

\begin{tabular}{|c|c|c|c|c|c|}
\hline Author(s) & Type participants & Baseline assessment & $\begin{array}{l}\text { Mean age at } \\
\text { baseline } \pm S D \\
\text { (range) }\end{array}$ & Outcome assessment & $\begin{array}{l}\text { Mean age at outcome } \pm \text { SD } \\
\text { (range) }\end{array}$ \\
\hline $\begin{array}{l}\text { Janssen et al., } \\
2009 \text { [45] }\end{array}$ & $\begin{array}{l}\text { PT infants (GA } \leq 32 w) \text { admitted to } \\
\text { NICU }\end{array}$ & $\begin{array}{l}\text { BSID-II PDI }<85 \text { and PDI }<90 \\
+ \text { subtest Behavior rating scale: } \\
\text { motor quality (Pc } \leq 10 \text { : Non- } \\
\text { optimal behavior, Pc11-25: } \\
\text { questionable behavior, Pc } \geq 26 \text { : } \\
\text { within normal limits behavior) }\end{array}$ & $\begin{array}{l}29 \mathrm{~m} \mathrm{CA} \\
( \pm 4,9)\end{array}$ & M-ABC-2-NL Pc $<15$ & $64 m \pm 2,3 m$ \\
\hline $\begin{array}{l}\text { Long et al., } \\
2016[48]\end{array}$ & $\begin{array}{l}\text { Children with Cardiac heart } \\
\text { disorder who underwent cardiac } \\
\text { surgery in the first } 2 \text { months of life }\end{array}$ & $\begin{array}{l}\text { AIMS Pc }<25 \\
\text { BSID-III }\end{array}$ & $\begin{array}{l}4 \mathrm{~m}, 8 \mathrm{~m}, \\
12 \mathrm{~m}, 16 \mathrm{~m} \\
2 \mathrm{y}\end{array}$ & BOT-2-Short Form Total Score SS <-1SD & $5 \mathrm{y} 8 \mathrm{~m} \pm 3 \mathrm{~m}$ \\
\hline $\begin{array}{l}\text { MacCobb } \\
\text { et al., } 2005 \\
\text { [40] }\end{array}$ & FT low-risk first-pregnancy infants & $\begin{array}{l}\text { NBAS subtest Muscle tone and } \\
\text { physical movements (= motor } \\
\text { scale) } \\
\text { BSID-I }\end{array}$ & $3 d$ and $21 d$ & BOT-MP Total Score SS $\leq 42$ & $9 y$ \\
\hline $\begin{array}{l}\text { Mazer et al., } \\
2010[42]\end{array}$ & $\begin{array}{l}\text { Mainly FT children with congenital } \\
\text { anomalies admitted to ICU without } \\
\text { major chromosomal or syndromal } \\
\text { abnormalities }\end{array}$ & BSID-I-NL PDI score < 84 & $\begin{array}{l}6 \mathrm{~m} \mathrm{CA}, 12 \mathrm{~m} \\
\mathrm{CA}, 24 \mathrm{~m}\end{array}$ & M-ABC-I-NL Pc $\leq 15$ & $5 y$ \\
\hline $\begin{array}{l}\text { Peyre et al., } \\
2018[54]\end{array}$ & Mainly FT children & $\begin{array}{l}\text { NEPSY-I: Copy Design Task ASQ-3 } \\
\text { subtests (1) Fine motor skills, (2) } \\
\text { Gross motor skills }\end{array}$ & $3 y$ & $\begin{array}{l}\text { NEPSY-I: Copy Design Task } \\
\text { ASQ-3 subtests ( } 1 \text { ) Fine motor skills, (2) } \\
\text { Gross motor skills }\end{array}$ & $5-6 y$ \\
\hline $\begin{array}{l}\text { Piek et al., } \\
2008[53]\end{array}$ & Low risk children & $\begin{array}{l}\text { ASQ subtests (1) Fine motor skills, } \\
\text { (2) Gross motor skills }\end{array}$ & $\begin{array}{l}4 \mathrm{~m}, 6 \mathrm{~m}, 8 \mathrm{~m} \\
12 \mathrm{~m}, 16 \mathrm{~m} \\
18 \mathrm{~m}, 20 \mathrm{~m} \\
24 \mathrm{~m}, 30 \mathrm{~m} \\
36 \mathrm{~m}, 48 \mathrm{~m}\end{array}$ & $\begin{array}{l}\text { MAND subtests: (1) Fine motor, (2) } \\
\text { Gross motor }\end{array}$ & $\begin{array}{l}8 \mathrm{y} 5 \mathrm{~m} \pm 1 \mathrm{y} 9 \mathrm{~m}(6 \mathrm{y} 0 \mathrm{~m}- \\
11 \mathrm{y} 6 \mathrm{~m})\end{array}$ \\
\hline $\begin{array}{l}\text { Roze et al., } \\
2010 \text { [36] }\end{array}$ & $\begin{array}{l}\text { Healthy FT singletons (overlapping } \\
\text { cohort study Hitzert et al., 204) }\end{array}$ & $\begin{array}{l}\text { GmA by Prechtl: quality of GMs } \\
\text { MOS } \\
\text { BSID-II-NL Pc }>5-\leq 15 \text { (suspect) } \\
\text { and Pc } \leq 5 \text { (abnormal) }\end{array}$ & $\begin{array}{l}12 \mathrm{w}(11- \\
13 \mathrm{w}) \\
18 \mathrm{~m} \\
(17 \mathrm{~m} 3 \mathrm{w}- \\
18 \mathrm{~m} 1 \mathrm{w})\end{array}$ & $\begin{array}{l}\text { M-ABC-I-NL Pc }>5-\leq 15 \text { (suspect) and } \\
\mathrm{Pc} \leq 5 \text { (abnormal) }\end{array}$ & $6 y 1 m(5 y 8 m-7 y-6 m)$ \\
\hline $\begin{array}{l}\text { Seme- } \\
\quad \text { Ciglenecki, } \\
2007 \text { [37] }\end{array}$ & High-risk PT children $(\mathrm{GA}<37 \mathrm{w})$ & GmA by Prechtl: Quality of GMs & $12 \mathrm{w} C A$ & $\begin{array}{l}\text { cMND by Modified Partial Touwen test } \\
\text { (Based on Examination of the child with } \\
\text { minor neurological dysfunction } 2 \text { nd } \\
\text { edition by Touwen, 1979) }\end{array}$ & 6y CA \\
\hline $\begin{array}{l}\text { Siegel, } 1983 \\
\quad[43]\end{array}$ & Mixed group PTs and FTs & BSID-I <-1SD & $\begin{array}{l}4 \mathrm{~m}, 8 \mathrm{~m}, \\
12 \mathrm{~m}, 18 \mathrm{~m}, \\
2 \mathrm{y}\end{array}$ & $\begin{array}{l}\text { McCarthy Scales of Children's Abilities } \\
\text { motor scale score }<85 \\
\text { Beery-VMI ratio-scores }\end{array}$ & $5 y$ \\
\hline $\begin{array}{l}\text { Siegel,1992 } \\
\quad[44]\end{array}$ & PT and matched FT children & $\begin{array}{l}\text { BSID-I Kohen-Raz score (Average } \\
\text { and below average) }\end{array}$ & $\begin{array}{l}4 \mathrm{~m}, 8 \mathrm{~m} \\
12 \mathrm{~m}, 18 \mathrm{~m} \\
2 \mathrm{y}\end{array}$ & Beery-VMI & $8 \mathrm{y}$ \\
\hline $\begin{array}{l}\text { Sustersic } \\
\text { et al., } 2012 \\
\text { [26] }\end{array}$ & PT infants & GmA by Prechtl: Quality of GMs & $\begin{array}{l}40 w \text { CA } \\
( \pm 5 \text { days })(0- \\
20 w), 3 m \text { CA } \\
(0-20 w)\end{array}$ & $\begin{array}{l}\text { Motor difficulties consistent with DCD } \\
\text { by M-ABC-I Pc }<15\end{array}$ & $5-6 y$ \\
\hline $\begin{array}{l}\text { Van Iersel } \\
\text { et al., } 2016 \\
\text { [38] }\end{array}$ & $\begin{array}{l}\text { FT infants with and without } \\
\text { difficulties at birth }\end{array}$ & $\begin{array}{l}\text { GmA by Hadders-Algra: Quality of } \\
\text { GMs }\end{array}$ & $\begin{array}{l}\text { Median 3w } \\
\text { CA }(38-47 w) \text {, } \\
\text { Median } 13 w \\
\text { CA }(48-58 w)\end{array}$ & $\begin{array}{l}\text { cMND by 'Neurological Examination of } \\
\text { the Child with Minor Neurological } \\
\text { Dysfunction' } \\
\text { M-ABC-I-NL Pc } \leq 15 \text { (total score) or } \\
\text { Pc } \leq 5 \text { (subtest) }\end{array}$ & $\begin{array}{l}\text { total group: median } 77,5 \mathrm{~m} \\
\text { (75-83); DBAT group: } \\
\text { median } 77 \mathrm{~m} \text { (75-83); } \\
\text { Non-DBAT group: median } \\
77,5 \mathrm{~m}(74-81)\end{array}$ \\
\hline $\begin{array}{l}\text { Wolthuis- } \\
\text { Stigter } \\
\text { et al., } 2017 \\
\text { [58] }\end{array}$ & $\begin{array}{l}\text { PTs }(\mathrm{GA}<36 \mathrm{w}) \text { without major } \\
\text { congenital defects and syndromes }\end{array}$ & NOMAS & $\begin{array}{l}\text { 37-40 PMA } \\
\text { (weekly) and } \\
42-50 w \text { PMA } \\
\text { (two- } \\
\text { weekly) }\end{array}$ & M-ABC-I-NL & $5 y 8 m(5 y 6 m-5 y 11 m)$ \\
\hline $\begin{array}{l}\text { Yuge et al., } \\
2011 \text { [39] }\end{array}$ & $\begin{array}{l}\text { PT and FT infants admitted to } \\
\text { hospital due high-risk perinatal } \\
\text { histories, abnormal findings } \\
\text { pediatric examination or parental } \\
\text { concerns }\end{array}$ & $\begin{array}{l}\text { GmA by Prechtl: Quality of GMs } \\
\text { MOS }\end{array}$ & $\begin{array}{l}\text { Median } 17 w \\
(9-21 w)\end{array}$ & $\begin{array}{l}\text { DCD (unspecified how diagnosis was } \\
\text { made) }\end{array}$ & $5 y$ \\
\hline
\end{tabular}

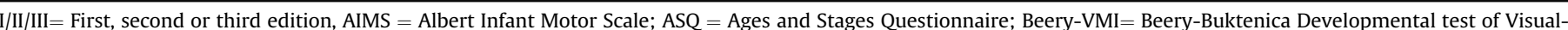

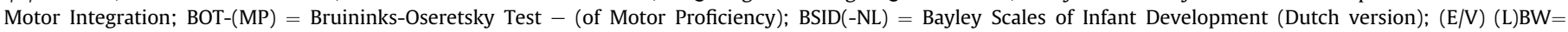

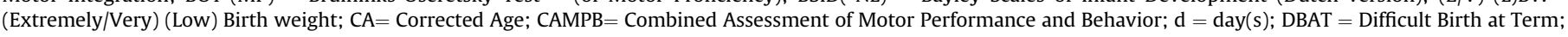

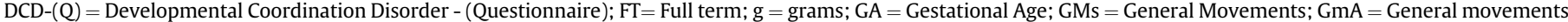

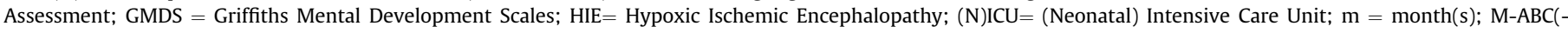

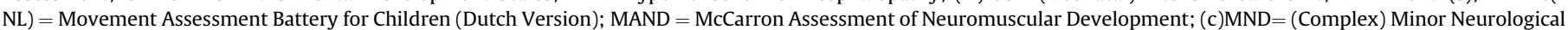

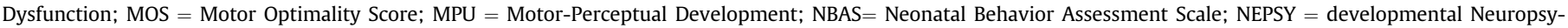

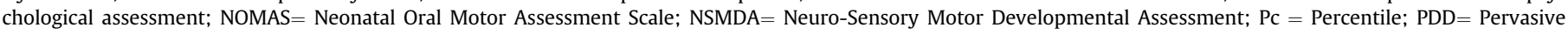

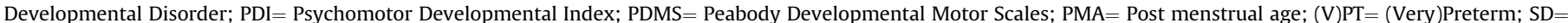

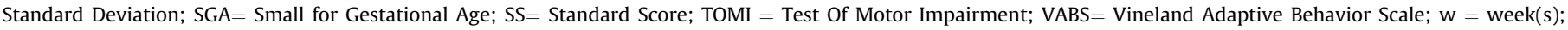
$\mathrm{y}=\operatorname{year}(\mathrm{s})$.

assessments. Due to relatively small sample sizes, regression analyses could not always be performed. Finally, the assessors were very rarely blinded to participant characteristics or previous assessment performances. The two poor-quality studies were the oldest included studies and only reported weak but significant correlations regarding the BSID-I. Concerning GmA, a slightly 
higher study quality was found for studies applying the GmA by Hadders-Algra's method compared to GmA by Prechtl's method. Nevertheless, no strong associations were observed between study quality and the strength of the reported predictive values.

\subsection{Predictive values grouped by outcome}

Outcome measures were defined as DCD, cMND and significant motor delays on a standardized assessment. Fig. 2 depicts the significance of prediction grouped by outcome.

\subsection{1. $D C D$}

The outcome measure was defined as DCD in only two studies $[39,57]$ and as probable DCD in another two studies [26,50]. Probable DCD was based on a score below the 15 th percentile on the M$A B C$ while also considering the exclusion criteria. A total of five baseline assessment instruments were used in these studies.

3.2.1.1. Combined Assessment of Motor Performance and Behavior ( $C A M P B)$. The included data of one study may suggest some evidence for the predictive value of CAMPB (Appendix B - Table 4). Neonatal Intensive Care Unit children with incoordination at $3 y$ seemed to be three to five times more likely to develop DCD at $6.5 \mathrm{y}$ [57]. DCD was defined as a score below the 15th percentile on the Test of Motor Impairment and a developmental delay in the area of Activities of daily living measured with the Motor-Perceptual Development (MPU). Additionally, these children did not have a general medical condition, pervasive developmental disorder, or mental retardation [57].
3.2.1.2. Griffiths Mental Development Scales (GMDS) - locomotor and eye-hand scale. The included data of one study may suggest some evidence for the predictive value of the motor subtests of the GMDS in relation to probable DCD (Appendix B - Table 5). Goyen et al. reported a significant association in preterm children scoring below the 43rd percentile only on the locomotor scale at 3y, but not on the eye-hand-scale [50].

3.2.1.3. Peabody Developmental Motor Scales (PDMS). The included data of one study may suggest some evidence for the predictive value of the PDMS in relation to probable DCD (Appendix B Table 13). Three-year-old children scoring below the 27th percentile on the fine motor scale or below the 41 st percentile on the gross motor scale were more likely to have probable DCD at 8 y [46].

3.2.1.4. General Movements Assessment (GmA) including Motor Optimality Score (MOS). Two methods are available within GmA: Prechtl's method (GmA-P) and Hadders-Algra's method (GmA-HA). Both methods offer the possibility to observe the quality of general movements over three age-periods namely preterm, writing, and fidgety age. The MOS is an addition to the standard GmA and consists of five subtests: 'Quality of fidgety movements', 'Age-adequacy', 'Presence and normality of individual movement patterns', 'Presence and normality of individual postural patterns', and 'Quality of the motor repertoire'.

The included data of two studies suggest no significant evidence for the predictive value of the quality of writing movements or fidgety movements in relation to DCD [39] or probable DCD [26], nor was there evidence for the predictive value of any of the MOS

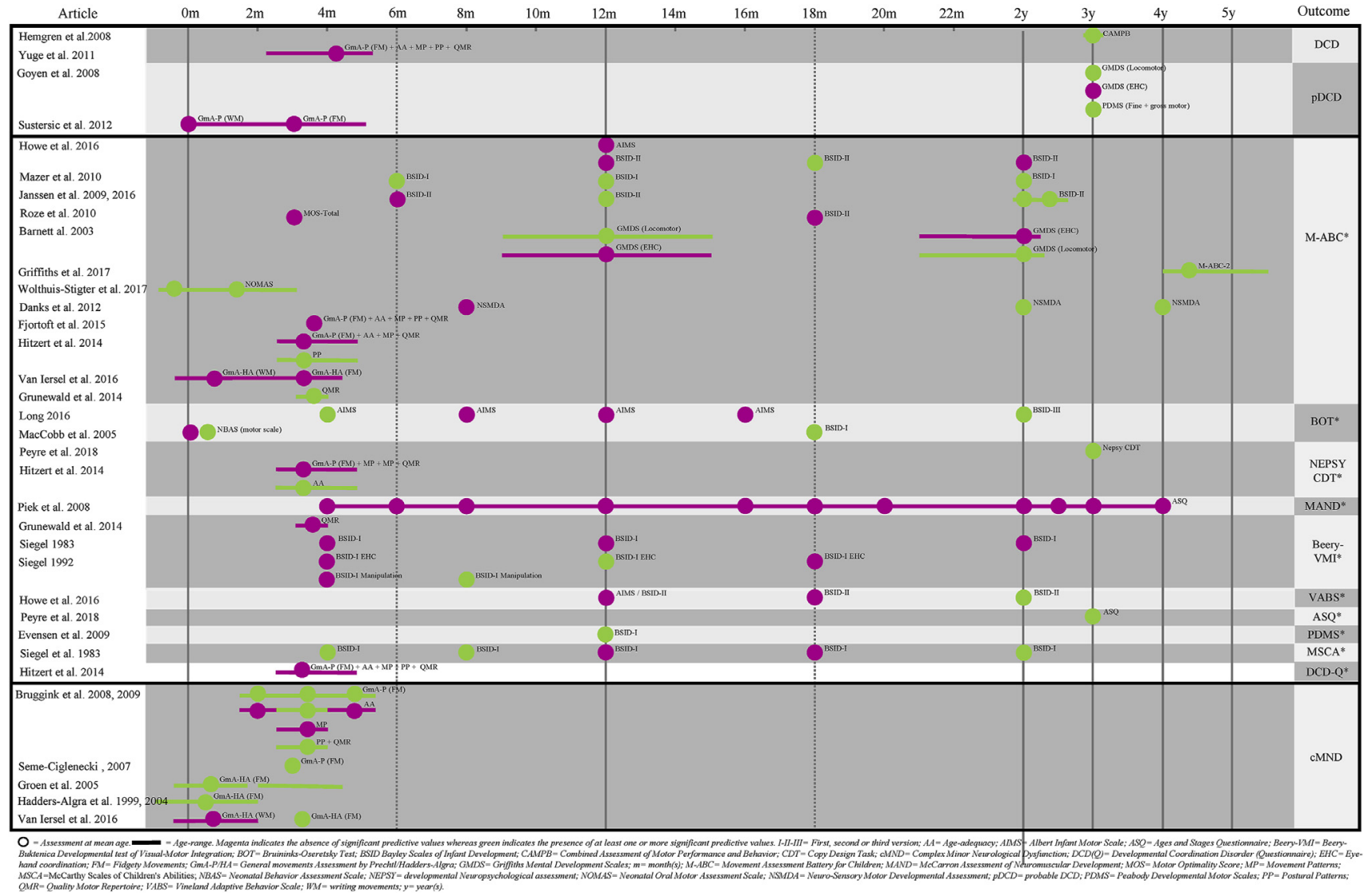

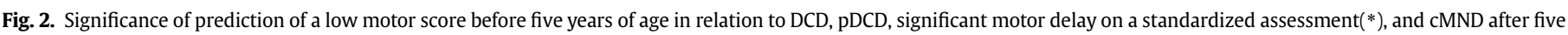
years of age. 
subscales in relation to DCD [39] (Appendix B - Tables 6-8).

\subsection{2. $C M N D$}

The outcome measure was defined as cMND in five studies $[27,28,30,32,33,37,38]$. A total of two baseline assessment instruments were used in these studies.

3.2.2.1. General Movements Assessment (GmA) including Motor Optimality Score (MOS). The included data of five studies suggest some evidence for the predictive value of the quality of general movements in relation to cMND (Appendix B - Tables 6-8).

Data on the quality of writing movements in relation to cMND was only available using Hadders-Algra's method. Results from all three studies applying GmA-HA indicate that children presenting with definitely abnormal writing movements were more likely to develop cMND [30,32,33,38]. However, confidence intervals of relative risks were very wide. Additionally, Van Iersel et al. reported an association between mildly/definitely abnormal writing movements and subsequent dysfunctional domains of 'Posture and tone' and 'Coordination' at $6 y$ after correcting for confounding variables [38]. Data on the quality of fidgety movements in relation to cMND was available in two studies using GmA-P [27,28,37] and three studies using GmA-HA [30,32,33,38]. In all studies but Van Iersel et al. [38], children with either abnormal/absent (GmA-P) [27,28,37] or definitely abnormal (GmA-HA) [30,32,33] fidgety movements appeared more likely to develop cMND. Yet again, confidence intervals were very wide. Furthermore, Van Iersel et al. reported an association between definitely abnormal fidgety movements and a subsequent dysfunctional domain of 'posture and tone' [38].

The included data of one study suggest only minor evidence for the predictive value of the MOS. Subtests 'Age-adequacy' and 'Quality of motor repertoire' at 11-16 weeks (w) seem related to cMND, but the 'Presence and normality of movement or postural patterns' are not $[27,28]$.

\subsubsection{Motor delays on a standardized assessment}

School-aged motor delays were described using the M-ABC in eleven studies [29,31,36,38,42,45-47,52,55,56,58], the BruininksOseretsky test (BOT) in two studies $[40,48]$, the NEPSY Copy Design Task in two studies [35,54], and the Beery - Developmental test of Visual-Motor Integration (Beery-VMI) in three studies [31,43,44]. Predictive values were available only once for the McCarron Assessment of Neuromuscular Development (MAND) [53], VABS [47], ASQ [54], PDMS [41], McCarthy Scales of Children's Abilities (MSCA) [43], and DCD-Questionnaire (DCD-Q) [35]. A total of ten baseline assessment instruments were used in these studies.

\subsubsection{Ages and Stages Questionnaire (ASQ) and NEPSY Copy Design} Task. The included data of two studies may suggest some evidence for the predictive value of ASQ scores, but not for ASQ trajectories (Appendix B - Table 1). In low-risk children of whom parents completed eleven ASQ's between the age of $4 \mathrm{~m}$ and $48 \mathrm{~m}$, no significant association could be withheld between the ASQ trajectories (i.e. age at which child reaches maximum performance, the maximum or minimum score, and the variance of ASQ scores) and MAND-scores at 6-11 y [53]. However, another study with a larger sample of low-risk children applying a novel edition of the ASQ described that children scoring below the 10th percentile on either the ASQ-3 (fine or gross motor subtest) or the NEPSY Copy Design Task at 3y, were three times more likely to retain the poor performances at 5-6 y [54]. Relative risk confidence intervals were narrow and regression analysis supported the predictive value of the NEPSY Copy Design Task, the ASQ-3-fine motor, and the ASQ-3-gross motor.
3.2.3.2. Alberta Infant Motor Scale (AIMS). The included data of two studies suggest conflicting evidence for the predictive value of AIMS (Appendix B - Table 2). Long et al. reported an association between AIMS-scores at $4 \mathrm{~m}$, but not $8 \mathrm{~m}, 12 \mathrm{~m}$, or $16 \mathrm{~m}$ and BOT-SF-scores in children with congenital heart disorders at 5-6 y [44]. Yet, in preterm low birth-weight (LBW) children, AIMS-scores at $12 \mathrm{~m}$ were not significantly associated with M-ABC-I total scores at $5 \mathrm{y}$ after correcting for confounding variables [47].

3.2.3.3. Bayley Scales of Infant Development (BSID). The included data may suggest some evidence for the predictive value of the Psychomotor Developmental Index (PDI) of the BSID first edition in five studies, the second edition in four studies, and the third edition in one study (Appendix B - Table 3).

Using the first version, significant associations were reported in all five studies although the studies of Siegel and MacCobb et al. could explain only a limited amount of the variance in school-aged performances on the BOT-MP [40], the MSCA motor scale [43], and the Beery-VMI [43,44]. PDI-scores at $6 \mathrm{~m}, 12 \mathrm{~m}$ and $24 \mathrm{~m}$ were strongly associated with lower M-ABC-I-scores at $5 \mathrm{y}$ in term-born children with congenital anomalies [42]. Additionally, Evensen et al. retrieved significant results in relation to PDMS-I-scores at 5y in the very low birth-weight group (VLBW) but not in the small-forgestational-age group or control group at $12 \mathrm{~m}$ [41].

Using the second version, significant associations were reported in three out of four studies. At $6 \mathrm{~m}$ no significant associations were withheld in relation to school-aged M-ABC-2-scores at 5 y [46]. At $12 \mathrm{~m}$ Janssen et al. [46] detected an elevated risk for poor M-ABCscores at $5 \mathrm{y}$ in preterm children while Howe et al. [47] did not. No significant association was withheld at $12 \mathrm{~m}$ or $18 \mathrm{~m}$ with the Chinese Vineland Adaptive Behavior Scales scores (VABS-C) at 5 y [47]. At $18 \mathrm{~m}$, Howe et al. [47] reported a significant association to $\mathrm{M}$ ABC-I-scores at 5y while Roze et al. [36], who studied low-risk children at 5-7y, did not. At $24 \mathrm{~m}$, preterm children with a low PDI were twice more likely to experience low M-ABC-2-scores at 5 y [46]. In contrast, Howe et al. only withheld significant results regarding VABS-C scores and not $\mathrm{M}-\mathrm{ABC}-\mathrm{I}-\mathrm{scores}$ [47]. At 29m, Janssen et al. [45] detected that preterm children with a low PDI were twice more likely to experience low M-ABC-2-scores at 5y. Demonstrating low motor quality on the behavior rating scale of the BSID-II also yielded significant higher odds.

Using the third version, the motor composite score at $2 \mathrm{y}$ explained $39 \%$ of the variance in BOT-SF-scores score at $5 \mathrm{y} 8 \mathrm{~m}$ in children with a congenital heart disorder, whereas the gross motor score explained $29 \%$ [44].

3.2.3.4. Griffiths Mental Development Scales (GMDS) - locomotor and eye-hand scale. The included data of one study may suggest some evidence for the predictive value of the motor subtests of the GMDS (Appendix B - Table 5). Full-term children with neonatal encephalopathy and low scores on GMDS locomotor scale at $1 \mathrm{y}$, but not $2 \mathrm{y}$, and eye-hand scale at $2 \mathrm{y}$, but not $1 \mathrm{y}$, seemed more likely to have low M-ABC-I-scores at 5-6 y [52]. However, confidence intervals were wide.

3.2.3.5. General Movements Assessment (GmA) including Motor Optimality Score (MOS). The included data of three studies suggest no evidence for the predictive value of the quality of general movements in relation to motor delays on a standardized assessment at school-age (Appendix B - Tables 6-8). The quality of writing movements by GmA-HA was not associated to M-ABC-I total score or any of its subtests at 6 y [38]. The quality of fidgety movements by GmA-P [29,35] or GmA-HA [38] was not associated to M-ABC-I [35], M-ABC-2 [29], NEPSY-II Copy Design Task [35] or DCD-Q [35]. 
The included data of four studies suggest only minor evidence for the predictive value of the MOS. The MOS total score explained merely $4 \%$ of the variance of the M-ABC-I scores at 5-7 y [36]. Nor 'age-adequacy' nor 'the presence and normality of movement patterns' were related to M-ABC-I at 5-7 y [35], M-ABC-2 at 10-11 y [29], or DCD-Q at 5-7 y [35]. Only Hitzert reported a weak association between 'age-adequacy', but not 'normality of movement patterns', and the NEPSY-II Copy Design Task at 5-7y. The 'presence and normality of postural patterns' could be related to M-ABC-I at 5-7 y [35], but not to M-ABC-2 at 10-11 y [29], NEPSY-II Copy Design Task at 5-7 y [35], or DCD-Q at 5-7 y [35,39]. 'The quality of the motor repertoire' was not related to M-ABC-I, NEPSY-II Copy Design Task, or DCD-Q at 5-7 y [35]. A significant association to M-ABC-2 total score at $10 \mathrm{y}$ was reported by Grunewaldt et al. [31], but not by Fjørtoft et al. at 10-11 y [29]. Additionally, Grunewaldt et al. reported a significant association with the M-ABC-2 subtest 'balance', but not with 'manual dexterity' or 'aiming and catching' [31].

3.2.3.6. Movement Assessment Battery for Children - second edition $(M-A B C-2)$. The included data of one study may suggest some evidence for the predictive value of $\mathrm{M}-\mathrm{ABC}-2$ in very preterm children (Appendix B - Table 9). After correcting for confounding and missing variables, a low score on the $\mathrm{M}-\mathrm{ABC}-2$ at $4.4 \mathrm{y}$ seemed to explain $50 \%$ of the variance of the M-ABC-2 scores at 7y11 m [55].

3.2.3.7. Neonatal Behavioral Assessment Scale (NBAS) - motor scale. The included data of one study suggest minor evidence for the predictive value of NBAS at 21 days (d), but not at $3 \mathrm{~d}$ (Appendix B Table 10) [40]. The NBAS at 21d explained $12 \%$ of variance on the balance subtest of the BOT-2 at 9y in low-risk children and $27 \%$ of the variance after correcting for the child's responsiveness and body motion.

3.2.3.8. Neurological, Sensory, Motor, Developmental Assessment (NSMDA). The included data of one study may suggest some evidence for the predictive value of NSMDA at $2 \mathrm{y}$ and $4 \mathrm{y}$, but not at $8 \mathrm{~m}$ in preterm extremely low birth weight (ELBW) children [56] (Appendix B - Table 11). Children with a low NSMDA score at $2 \mathrm{y}$ or $4 y$ were twice more likely to have low M-ABC-I-scores at 11-13y.

3.2.3.9. Neonatal Oral-Motor Assessment Scale (NOMAS). The included data of one study may suggest some evidence for the predictive value of NOMAS (Appendix B - Table 12). WolthuisStigter et al. indicated that a normal sucking pattern in a mixed group of children at 50w post-menstrual age (PMA), but not 40w PMA, was associated with better M-ABC-I-scores 5y [58]. In contrast, children with prolonged definitely abnormal sucking patterns were more likely to experience low M-ABC-I-scores. Furthermore, moderate positive correlations were reported between several items of NOMAS and the balance subtest of M-ABC-I.

\subsection{Predictive values grouped by age at baseline assessment}

As depicted in Fig. 2, predictive results vary greatly in the first two years of life. Before $12 \mathrm{~m}$, the GmA and MOS subtests seem associated to cMND [27,28,30,32,33,38], but not to DCD [26,39] or motor delays on standardized motor assessments [27-29,35,36,38]. NOMAS [48,58] was related to school-aged motor delay while inconsistent results were obtained regarding AIMS [47], NBAS motor scale [40], and BSID [42-46]. As children age, predictive values tend to improve. Associations between BSID and motor outcome demonstrate significant results in four $[41,42,44-47]$ out of six studies $[43,47]$ at $12 \mathrm{~m}$, two $[40,47]$ out of five studies [36,43] at $18 \mathrm{~m}$ and in all five studies at 2 y [42-48]. Similarly, the NSMDA was only associated to school-aged M-ABC-I- scores at $2 \mathrm{y}$ and $4 \mathrm{y}$, but not at $8 \mathrm{~m} \mathrm{[56].} \mathrm{At} \mathrm{3y,} \mathrm{an} \mathrm{association} \mathrm{was}$ reported between (probable) DCD and the scores on the PDMS [50], the GMDS Locomotor scale [50], and the CAMPB [57]. Furthermore, NEPSY Copy Design task and ASQ [54] seem valuable in predicting significant motor delays. Lastly, M-ABC-2 between 4.5 and $5.5 y$ likewise showed promising results in predicting school-aged motor delays on standardized motor tests [55].

\subsection{Summary}

The GmA seems to be of modest value in the prediction of cMND, but not for other motor outcomes or (probable) DCD. Regarding the prediction of school-aged motor problems, other than CMND, the ratio sensitivity/specificity was best in BSID-I at $1 \mathrm{y}$ (67/100) [41] and M-ABC-2 at mean age of 4.4y (72/93) [55] with the latter presented in a higher quality study. Sensitivity was found highest for PDMS at $3 y$ (94\%) [50] and BISD-II at 1y (93\%) [46], while specificity was found highest in BSID-I at 1y (100\%) [41] and GMDS locomotor scale at $1 \mathrm{y}(100 \%)$ [52]. The ratio NPV/PPV was best in BSID-I at $1 \mathrm{y}(100 / 93)$ [41] and GMDS Locomotor scale at $1 \mathrm{y}(100 / 80)$ [52] with the latter presented in a higher quality study. PPV was found highest for BSID-I at 1y (100\%) [41] and GMDS locomotor scale at $1 \mathrm{y}(100 \%)$ [52], while NPV was found highest in CAMPB at $3 y$ (96\%) [57], M-ABC-2 at mean age of 4.4y (93\%) [55] and BSID-I at 1y (93\%) [41]. The ability to detect motor problems seems to be elevated in high-risk groups and increases as children grow older. The number of studies specifically investigating associations between early motor development and subsequent DCD is very limited.

\section{Discussion}

\subsection{Main findings}

This review aimed to summarize which standardized motor assessments before the age of five are available to accurately predict DCD, cMND, and significant motor delays on a standardized assessment in children aged five to twelve years. The majority of early motor assessments included in this review seem to have some predictive value for school-aged motor problems. This finding indicates that motor problems relating to DCD may already be detectable early in life. Nevertheless, no assessment instrument reached the recommended $80 \%$ sensitivity and specificity [60] and merely four studies specifically reported (probable) DCD as outcome. Together with the large clinical heterogeneity and the great variety in assessment instruments, applied cut-offs, reported terminology and statistical methods, this makes it very difficult to compare studies and draw solid conclusions.

Importantly, only two studies reported DCD as a distinct outcome measure. The motor assessments suggested by the EACD to aid in operationalizing criterion A of the DSM-5 [1] are M-ABC-2 and BOT-2 whereas the DCD-Q has been suggested for criterion $B$ [2]. However, diagnostic criteria of DCD are still open for interpretation and are not always well-described in research settings. Only Hemgren et al. [57] considered all diagnostic criteria, while Yuge et al. [39] did not elaborate on how the children were diagnosed. Another two studies reported 'probable DCD' based on a total M-ABC score below the $15^{\text {th }}$ percentile $[26,50]$. Although both studies excluded children with other disorders possibly responsible for the motor delay (criterion D), they did not account for criterion B. These four studies suggest some minor evidence that the CAMPB at 3 y [53], the GMDS locomotor scale at 3 y [46], and the PDMS at 3 y [46] may have some predictive value for (probable) DCD, while the GmA and MOS do not [26,39]. However more longitudinal studies starting in infancy with well-applied diagnostic criteria for 
DCD or clinical diagnoses are necessary.

MND is an often reoccurring outcome measure in the included studies. Although a relation has been described between MND and DCD, the mechanisms for this relationship are still unclear. We cannot assume that all children with cMND fulfill the diagnostic criteria for DCD, although an important part of them will [14]. This review adds to that discussion given that $\mathrm{GmA}$ and MOS appeared associated to CMND, but not to DCD or low scores on the M-ABC-2, the most widely used assessment instrument in the DCD field. As GmA and cMND focus on central nervous integrity, the M-ABC-2 evaluates several specific functional motor skills, which might explain the lack of association between GmA and M-ABC-2 [36]. Prediction of school-aged cMND was solely investigated using GmA or MOS in early infancy. Using Prechtl's method, absent and abnormal fidgety movements seemed related to cMND whereas in Hadders-Algra's method, definitely abnormal fidgety movements seemed associated to cMND. Although confidence intervals were quite large, prompting for careful interpretation, this finding is somewhat surprising, as abnormal fidgety movements have previously been associated in a greater extent with CP [38]. As this review exclusively included data of children without $\mathrm{CP}$, this might have shifted the observed associations. The results of this review indicate the presence of a certain association between GmA and MOS in early infancy and school-aged cMND, albeit more research is necessary to explore the concept of MND and its relation to DCD.

The majority of the included studies investigated the association between early motor assessment and significant motor delays at school-age measured with a standardized assessment. In the studies that provided sufficient data to calculate sensitivity and specificity, the BSID-I and BSID-II showed the highest sensitivity [46] and specificity [41] respectively. The ratio sensitivity/specificity is also highest in the BSID-I [41] and the M-ABC-2 [55]. The only included study applying the BSID-III was able to explain a large part of the variance in school-aged BOT-2-scores, but did not offer data to calculate sensitivity and specificity. Still, this study comprised children with congenital heart disorders, so generalization of these findings is limited as it possible that the experienced motor problems may be explained by other factors. Nevertheless, as the first editions of the BSID do suggest some promising results and strong correlations have been reported between the BSID-II-PDI and Bayley-III motor composite score [61], BSID-III might also be valuable in detecting early motor problems starting at the age of $1 \mathrm{~m}$. Recently, the new BSID-IV was published, but no information is available yet on its predictive value. The MABC-2 is a suitable assessment instrument starting at $3 \mathrm{y} \mathrm{[55].}$ However, the relatively good predictive values were not surprising as it was the same test which was used, only a few years later. The GmA did not seem associated to motor delays at school-age in this group of non-CP children. A 2011 systematic review proposed that $\mathrm{GmA}$ might not sufficiently tap into the areas necessary to detect more subtle impairments [62]. Some suggest that motor delays in this group of children may not be apparent this early in life when motor demands are less complex. Nevertheless, NOMAS at 37-50w PMA [58], the NBAS at $21 \mathrm{~d}$ [40], and the AIMS at $4 \mathrm{~m}$ could all be related to school-aged motor performance.

Remarkably, only one parental questionnaire, the ASQ was applied before $5 y$. Piek et al. was unable to detect significant associations between ASQ-trajectories and a subsequent lower MAND-score at school-age [53], while Peyre did reported significant associations between ASQ at 3y and ASQ at 5-6y. This association is not surprising as the outcome measure was the same. Although the ASQ is a reliable and valid questionnaire, doubts about the reliability of parental questionnaires in children with DCD have been reported [63]. It would be interesting to investigate the predictive value of parental questionnaires specifically designed for detecting DCD before $5 y$ such as the Little-DCDQuestionnaire. More research is necessary to clarify the relationship between infant motor development and motor delay at schoolage.

It is clear that prediction of school-aged motor outcome poses a complex and difficult challenge. Accordingly, EACD-guidelines suggest to only diagnose DCD before $5 y$ of age when repeated assessments indicate motor problems. Many studies report high variability in longitudinal motor performance as a typical developmental trait and emphasize that one should cautiously interpret results from a single assessment in time [64-66]. Indeed, manifold low motor scores strengthen the predictive values of later developmental outcomes [47,51,67]. A combination of diverse assessments or sequential screening [68] might be extremely valuable in improving the predictive accuracy. Hemgren et al. reported better predictive values for DCD when combining both motor incoordination and inattention [57]. Additionally, Spittle et al. demonstrated a consistently higher accuracy when combining results of the AIMS and the NSMDA in the prediction of low M-ABC scores at 4 y [67].

As children grow older, predictive values tend to improve. This may be explained by various reasons. Firstly, a possible catch-up effect should be considered, especially in very preterm children during the first year of infancy [69]. Secondly, Goyen et al. suggest that children may present with more motor problems as their age increases because of the augmented complexity of test items [49]. Therefore, children might grow into motor problems due to higher demands of complex neurological functioning [70]. Finally, one has also to keep in mind that prediction is greatly influenced by the psychometric quality of the chosen instrument. Moreover, test results are snapshots of the child's development and may be influenced by the child's mood, motivation, and health status. Hence, besides using a valid and reliable assessment instrument, clinical judgment remains important when assessing children [71]. The quality of movement, functional motor success, and parental concerns should also be considered [51,72].

\subsection{Study strengths and limitations}

Many studies were excluded since data was not provided for non-CP children. Although, Williams et al. [73] claim some sort of continuum between DCD and CP, they are two distinct conditions. It would be beneficial if studies would report separate results for these two groups as the prediction of $\mathrm{CP}$ is more straightforward and the inclusion of children with $\mathrm{CP}$ might cause inflated predictive values. We contacted authors to supply additional information on the non-CP group, which we believe is a vast benefit of this systematic review as novel data of this subgroup may provide new insights. A publication bias may be present as only published fulltext were included. Additionally, we did not account for known risk-factors which may possibly influence the results. Although some studies accounted for confounding variables, this was not always the case. In addition, many studies included very specific populations and small sample sizes, limiting comparison and generalization of the results. Nevertheless, given the vast heterogeneity, a meta-analysis to account for these confounders was not appropriate. Many studies applied diverse assessment instruments over time, thus the inherent differences between assessment instruments may also explain part of the variance between scores at baseline and outcome assessment. However, these assessment instruments are widely accepted tools to identify children with motor problems and are applied similarly in follow-up programs. The vast majority of included studies assessed motor delay at school-age used a cut-off score of at least one standard deviation below the mean on the $\mathrm{M}-\mathrm{ABC}$ or the BOT. In research settings, children are 
often catalogued into the probable DCD-group based on a low motor score (criterion A). So, although these children were not formally examined for DCD and detected motor problems may be due to other reasons, it seemed valuable to include them in our review.

Given the extensive searches in seven different databases, we believe we have identified all of the existing literature. Yet ten studies were identified by applying backward and forward referencing, indicating we might have overlooked some studies. As we only included outcome assessments after the age of $5 \mathrm{y}$, we might have missed important studies reporting predictive values for younger children.

Furthermore, the risk of bias of the included studies introduces another possible limitation. Few assessors were blinded to participant characteristics or previous assessment performances, and the majority of studies did not account for confounding variables, nor did they elaborate sufficiently on the psychometric properties of the applied assessment instruments.

\subsection{Implication for clinical practice}

Motor assessments before the age of five are valuable in detecting DCD, cMND, and motor delay. Repeated assessments over multiple developmental domains and time periods seem to enhance predictive accuracy. Based on the compiled data, we carefully suggest that in infancy, GmA may be useful in detecting CMND, but not DCD. As the previous editions of the BSID did suggest promising results, its novel edition might also be valuable in detecting motor delay. The $\mathrm{M}-\mathrm{ABC}-2$ seems valuable starting at $3 \mathrm{y}$. More research is necessary to confirm these hypotheses.

\section{Conclusion}

As DCD is a heterogeneous disorder with many clinical appearances, the early developmental process may also vary considerably between individuals. No motor assessment instrument will ever be able to accurately identify all children with motor delay or DCD at school-age. At or before $2 \mathrm{y}$ of age, the BSID, motor subtests of GMDS, NOMAS and NSMDA seem valuable. More research is needed to determine the most appropriate assessment instrument in this age-group. Starting at 3y, the PDMS, motor subtests GMDS, NSDMA, M-ABC-2 and CAMPB show promising results. They seem particularly valuable in predicting which children will not develop DCD, cMND or motor delays. To conclude, the DCD-field necessitates more high-quality longitudinal studies including diverse assessments at multiple ages, starting in infancy, with attention to risk-factors, quality of movement, parental concerns and applying well-defined diagnostic criteria of DCD.

\section{Funding}

This research did not receive any specific grant from funding agencies in the public, commercial, or not-for-profit sectors.

\section{Declaration of competing interest}

The authors declare that they have no known competing financial interests or personal relationships that could have appeared to influence the work reported in this paper.

\section{Appendix A. Supplementary data}

Supplementary data to this article can be found online at https://doi.org/10.1016/j.ejpn.2020.12.003.

\section{References}

[1] AmericanPsychiatricAssociation, Diagnostic and Statistical Manual of Mental Disorders (DSM-5(R), American Psychiatric Pub, 2013.

[2] R. Blank, et al., International clinical practice recommendations on the definition, diagnosis, assessment, intervention, and psychosocial aspects of developmental coordination disorder, Dev. Med. Child Neurol. 61 (2019) 242-285, https://doi.org/10.1111/dmcn.14132.

[3] K. Ahern, Developmental coordination disorder: validation of a qualitative analysis using statistical factor Analysis, Int. J. Qual. Methods 1 (2016) 70-82, https://doi.org/10.1177/160940690200100305.

[4] C. Missiuna, S. Moll, M. Law, S. King, G. King, Mysteries and mazes: parents' experiences of children with developmental coordination disorder, Can. J. Occup. Ther. 73 (2006) 7-17, https://doi.org/10.2182/cjot.05.0010.

[5] H.W. Kilbride, G.P. Aylward, B. Carter, What are we measuring as outcome? Looking beyond neurodevelopmental impairment, Clin. Perinatol. 45 (2018) 467-484, https://doi.org/10.1016/j.clp.2018.05.008.

[6] E.K. Webster, C.K. Martin, A.E. Staiano, Fundamental motor skills, screentime, and physical activity in preschoolers, J. Sport Health Sci. 8 (2019) 114-121, https://doi.org/10.1016/j.jshs.2018.11.006.

[7] H.C. Karras, D.N. Morin, K. Gill, S. Izadi-Najafabadi, J.G. Zwicker, Healthrelated quality of life of children with developmental coordination disorder, Dev. Disabil. Res. Rev. 84 (2019) 85-95, https://doi.org/10.1016/ j.ridd.2018.05.012.

[8] R.A. Lima, A. Bugge, A.K. Ersbøll, D.F. Stodden, L.B. Andersen, The longitudinal relationship between motor competence and measures of fatness and fitness from childhood into adolescence, J. Pediatr. 95 (2019) 482-488, https:// doi.org/10.1016/j.jped.2018.02.010.

[9] K.M. Heuser, J. Jaekel, D. Wolke, Origins and predictors of friendships in 6- to 8-year-old children born at neonatal risk, J. Pediatr. 193 (2018) 93-101, https://doi.org/10.1016/j.jpeds.2017.09.072, e105.

[10] S. Omer, A.M. Jijon, H.C. Leonard, Research Review: internalising symptoms in developmental coordination disorder: a systematic review and metaanalysis, JCPP (J. Child Psychol. Psychiatry) 60 (2019) 606-621, https:// doi.org/10.1111/jcpp.13001.

[11] S. Izadi-Najafabadi, N. Ryan, G. Ghafooripoor, K. Gill, J.G. Zwicker, Participation of children with developmental coordination disorder, Res. Dev. Disabil. 84 (2019) 75-84, https://doi.org/10.1016/j.ridd.2018.05.011.

[12] M.A.M. Cleaton, P.K. Lorgelly, A. Kirby, Developmental coordination disorder: the impact on the family, Qual. Life Res. 28 (2019) 925-934.

[13] J. Hunt, J. Zwicker, E. Godecke, A. Raynor, Awareness and knowledge of developmental coordination disorder: a survey of caregivers, teachers, allied health professionals and medical professionals in Australia, Child Care Health Dev (2020) 1-10.

[14] F. Ferrari, et al., Preterm birth and developmental problems in the preschool age. Part I: minor motor problems, J. Matern. Fetal Neonatal Med. 25 (2012) 2154-2159, https://doi.org/10.3109/14767058.2012.696164.

[15] M. Hadders-Algra, Two distinct forms of minor neurological dysfunction: perspectives emerging from a review of data of the Groningen Perinatal Project, Dev. Med. Child Neurol. 44 (2002) 561-571.

[16] L.H. Peters, C.G. Maathuis, M. Hadders-Algra, Limited motor performance and minor neurological dysfunction at school age, Acta Paediatr. 100 (2011) $271-278$.

[17] C. Missiuna, B.R. Gaines, N. Pollock, Recognizing and referring children at risk for developmental coordination disorder: role of the speech-language pathologist, Int. J. Speech Lang. 26 (2002) 172-179.

[18] D. Sugden, A. Kirby, C. Dunford, Movement difficulties in children: developmental coordination disorder, Int. J. Disabil. Dev. Educ. 55 (2008) 93-96, https://doi.org/10.1080/10349120802033360.

[19] M.J. De Kleine, M.W. Nijhuis-Van Der Sanden, A. Lya Den Ouden, Is paediatric assessment of motor development of very preterm and low-birthweight children appropriate? Acta Paediatr. 95 (2006) 1202-1208, https://doi.org/ 10.1080/08035250500525301.

[20] Y. Noble, R. Boyd, Neonatal assessments for the preterm infant up to 4 months corrected age: a systematic review, Dev. Med. Child Neurol. 54 (2012) 129-139, https://doi.org/10.1111/j.1469-8749.2010.03903.x.

[21] A.J. Spittle, L.W. Doyle, R.N. Boyd, A systematic review of the clinimetric properties of neuromotor assessments for preterm infants during the first year of life, Dev. Med. Child Neurol. 50 (2008) 254-266, https://doi.org/ 10.1111/j.1469-8749.2008.02025.x.

[22] K.R. Heineman, M. Hadders-Algra, Evaluation of neuromotor function in infancy-A systematic review of available methods, J. Dev. Behav. Pediatr. 29 (2008) 315-323, https://doi.org/10.1097/DBP.0b013e318182a4ea.

[23] A. Liberati, et al., The PRISMA statement for reporting systematic reviews and meta-analyses of studies that evaluate health care interventions: explanation and elaboration, PLoS Med. 6 (2009), https://doi.org/10.1016/ j.jclinepi.2009.06.006.

[24] R. Blank, et al., International clinical practice recommendations on the definition, diagnosis, assessment, intervention, and psychosocial aspects of developmental coordination disorder, Dev. Med. Child Neurol. 61 (2019) 242-285, https://doi.org/10.1111/dmcn.14132.

[25] L. National Heart, Blood Institute. Quality Assessment Tool for Observational Cohort and Cross-Sectional Studies, National Institutes of Health, Department of Health and Human Services, Bethesda, 2014. 
[26] B. Sustersic, K. Sustar, D. Paro-Panjan, General movements of preterm infants in relation to their motor competence between 5 and 6 years, Eur. J. Paediatr. Neurol. 16 (2012) 724-729, https://doi.org/10.1016/j.ejpn.2012.05.008.

[27] J.L. Bruggink, et al., Quantitative aspects of the early motor repertoire in preterm infants: do they predict minor neurological dysfunction at school age? Early Hum. Dev. 85 (2009) 25-36.

[28] J.L. Bruggink, et al., The quality of the early motor repertoire in preterm infants predicts minor neurologic dysfunction at school age, J. Pediatr. 153 (2008) 32-39.

[29] T. Fjørtoft, et al., Adaptive behavior in 10-11 year old children born preterm with a very low birth weight (VLBW), Eur. J. Paediatr. Neurol. 19 (2015) 162-169, https://doi.org/10.1016/j.ejpn.2014.11.006.

[30] S.E. Groen, A.C. de Blecourt, K. Postema, M. Hadders-Algra, General movements in early infancy predict neuromotor development at 9 to 12 years of age, Dev. Med. Child Neurol. 47 (2005) 731-738, https://doi.org/10.1017/ S0012162205001544.

[31] K.H. Grunewaldt, et al., Follow-up at age 10 years in ELBW children - functional outcome, brain morphology and results from motor assessments in infancy, Early Hum. Dev. 90 (2014) 571-578, https://doi.org/10.1016/ j.earlhumdev.2014.07.005.

[32] M. Hadders-Algra, A.M.C. Groothuis, Quality of general movements in infancy is related to neurological dysfunction, ADHD, and aggressive behaviour, Dev. Med. Child Neurol. 41 (1999) 381-391, https://doi.org/10.1111/ j.1469-8749.1999.tb00623.x.

[33] M. Hadders-Algra, et al., Quality of general movements and the development of minor neurological dysfunction at toddler and school age, Clin. Rehabil. 18 (2004) 287-299, https://doi.org/10.1191/0269215504cr730oa.

[34] E.G. Hamer, A.F. Bos, M. Hadders-Algra, Specific characteristics of abnormal general movements are associated with functional outcome at school age, Early Hum. Dev. 95 (2016) 9-13, https://doi.org/10.1016/ j.earlhumdev.2016.01.019.

[35] M.M. Hitzert, E. Roze, K.N. Van Braeckel, A.F. Bos, Motor development in 3month-old healthy term-born infants is associated with cognitive and behavioural outcomes at early school age, Dev. Med. Child Neurol. 56 (2014) 869-876.

[36] E. Roze, et al., Developmental trajectories from birth to school age in healthy term-born children, Pediatrics 126 (2010) e1134-e1142, https://doi.org/ 10.1542/peds.2010-0698.

[37] P. Seme-Ciglenecki, Predictive values of cranial ultrasound and assessment of general movements for neurological development of preterm infants in the Maribor region of Slovenia, Wien Klin. Wochenschr. 119 (2007) 490-496, https://doi.org/10.1007/s00508-007-0839-7.

[38] P.A.M. van Iersel, S.C.M. Bakker, A.J.H. Jonker, M. Hadders-Algra, Does general movements quality in term infants predict cerebral palsy and milder forms of limited mobility at 6 years? Dev. Med. Child Neurol. 58 (2016) 1310-1316, https://doi.org/10.1111/dmcn.13228.

[39] M. Yuge, et al., Movements and postures of infants aged 3 to 5 months: to what extent is their optimality related to perinatal events and to the neurological outcome? Early Hum. Dev. 87 (2011) 231-237, https://doi.org/ 10.1016/j.earlhumdev.2010.12.046.

[40] S. MacCobb, S. Greene, K. Nugent, P. O'Mahony, Measurement and prediction of motor proficiency in children using Bayley infant scales and the BruininksOseretsky test, Phys. Occup. Ther. Pediatr. 25 (2005) 59-79, https://doi.org/ 10.1080/J006v25n01_05.

[41] K.A.I. Evensen, J. Skranes, A.M. Brubakk, T. Vik, Predictive value of early motor evaluation in preterm very low birth weight and term small for gestational age children, Early Hum. Dev. 85 (2009) 511-518, https:// doi.org/10.1016/j.earlhumdev.2009.04.007.

[42] P. Mazer, et al., Early developmental assessment of children with major noncardiac congenital anomalies predicts development at the age of 5 years, Dev. Med. Child Neurol. 52 (2010) 1154-1159.

[43] L.S. Siegel, Correction for prematurity and its consequences for the assessment of the very low birth weight infant, Child Dev. 54 (1983) 1176-1188.

[44] L.S. Siegel, Infant motor, cognitive, and language behaviors as predictors of achievement at school age, Adv. Infancy Res. 7 (1992) 227-237.

[45] A.J. Janssen, et al., A model to predict motor performance in preterm infants at 5 years, Early Hum. Dev. 85 (2009) 599-604, https://doi.org/10.1016/ j.earlhumdev.2009.07.001.

[46] A.J. Janssen, et al., High variability of individual longitudinal motor performance over five years in very preterm infants, Res. Dev. Disabil. 59 (2016) 306-317, https://doi.org/10.1016/j.ridd.2016.09.017.

[47] T.-H. Howe, C.-F. Sheu, Y.-W. Hsu, T.-N. Wang, L. Wang, Predicting neurodevelopmental outcomes at preschool age for children with very low birth weight, Res. Dev. Disabil. 48 (2016) 231-241, https://doi.org/10.1016/ j.ridd.2015.11.003.

[48] S.H. Long, B.J. Eldridge, S.R. Harris, M.M. Cheung, Motor skills of 5-year-old children who underwent early cardiac surgery, Cardiol. Young 26 (2015) 650-657, https://doi.org/10.1017/S1047951115000797.

[49] T.-A. Goyen, K. Lui, Longitudinal motor development of "apparently normal" high-risk infants at 18 months, 3 and 5 years, Early Hum. Dev. 70 (2002) 103-115, https://doi.org/10.1016/S0378-3782(02)00094-4.

[50] T.A. Goyen, K. Lui, Developmental coordination disorder in "apparently normal" schoolchildren born extremely preterm, Arch. Dis. Child. 94 (2008) 298-302, https://doi.org/10.1136/adc.2007.134692.

[51] K. Eldred, J. Darrah, Using cluster analysis to interpret the variability of gross motor scores of children with typical development, Phys. Ther. 90 (2010) 1510-1518, https://doi.org/10.2522/ptj.20090308.

[52] A.L. Barnett, et al., Can the Griffiths scales predict neuromotor and perceptualmotor impairment in term infants with neonatal encephalopathy? Arch. Dis. Child. 89 (2003) 637-643, https://doi.org/10.1136/adc.2002.019349.

[53] J.P. Piek, L. Dawson, L.M. Smith, N. Gasson, The role of early fine and gross motor development on later motor and cognitive ability, Hum. Mov. Sci. 27 (2008) 668-681.

[54] H. Peyre, et al., Developmental trajectories of motor skills during the preschool period, Eur. Child Adolesc. Psychiatr. (2019) 1-14, https://doi.org/ 10.1007/s00787-019-01311-x.

[55] A. Griffiths, et al., Predictive value of the movement assessment battery for children-second edition at 4 years, for motor impairment at 8 years in children born preterm, Dev. Med. Child Neurol. 59 (2017) 490-496.

[56] M. Danks, et al., The long-term predictive validity of early motor development in "apparently normal” ELBW survivors, Early Hum. Dev, 88 (2012) 637-641.

[57] E. Hemgren, K. Persson, Deficits in motor co-ordination and attention at 3 years of age predict motor deviations in 6.5-year-old children who needed neonatal intensive care, Child Care Health Dev. 35 (2009) 120-129, https:/ doi.org/10.1111/j.1365-2214.2008.00896.x.

[58] M.I. Wolthuis-Stigter, et al., Sucking behaviour in infants born preterm and developmental outcomes at primary school age, Dev. Med. Child Neurol. 59 (2017) 871-877, https://doi.org/10.1111/dmcn.13438.

[59] Eldred, K. \& Darrah, J. Using cluster analysis to interpret the variability of gross motor scores of children with typical development. Phys. Ther. 90, 1510-1518, doi:10.2522/ptj.20090308.

[60] F.P. Glascoe, Screening for developmental and behavioral problems, Ment Retard. Dev. Disabil. Res. Rev. 11 (2005) 173-179, https://doi.org/10.1002/ mrdd.20068.

[61] N. Bayley, in: Bayley Scales of Infant and Toddler Development, third ed. Harcourt assessment, 2006.

[62] V. Darsaklis, L.M. Snider, A. Majnemer, B. Mazer, Predictive validity of prechtl's method on the qualitative assessment of general movements: a systematic review of the evidence, Dev. Med. Child Neurol. 53 (2011) 896-906, https://doi.org/10.1111/j.1469-8749.2011.04017.x.

[63] G. Roberts, et al., Developmental coordination disorder in geographic cohorts of 8-year-old children born extremely preterm or extremely low birthweight in the 1990s, Dev. Med. Child Neurol. 53 (2011) 55-60, https://doi.org/ 10.1111/j.1469-8749.2010.03779.x.

[64] P. Van Geert, M. Van Dijk, Focus on variability: new tools to study intraindividual variability in developmental data, Infant Behav. Dev. 25 (2002) 340-374, https://doi.org/10.1016/S0163-6383(02)00140-6.

[65] R.S. Siegler, Variability and infant development, Infant Behav. Dev. 25 (2002) 550-557, https://doi.org/10.1016/S0163-6383(02)00150-9.

[66] J. Darrah, M. Hodge, J. Magill-Evans, G. Kembhavi, Stability of serial assessments of motor and communication abilities in typically developing infants-implications for screening, Early Hum. Dev. 72 (2003) 97-110, https://doi.org/10.1016/s0378-3782(03)00027-6.

[67] A.J. Spittle, et al., Accuracy of two motor assessments during the first year of life in preterm infants for predicting motor outcome at preschool age, PloS One 10 (2015), e0125854, https://doi.org/10.1371/journal.pone.0125854.

[68] N. Rawat, Psychological Assessment, MD Publications Pvt. Ltd., 2006.

[69] J.F. de Kieviet, J.P. Piek, C.S. Aarnoudse-Moens, J. Oosterlaan, Motor development in very preterm and very low-birth-weight children from birth to adolescence: a meta-analysis, J. Am. Med. Assoc. 302 (2009) 2235-2242. https://doi.org/10.1001/jama.2009.1708.

[70] M. Hadders-Algra, Evaluation of motor function in young infants by means of the assessment of general movements: a review, Pediatr. Phys. Ther. 13 (2001) 27-36, https://doi.org/10.1097/00001577-200113010-00005.

[71] B. Provost, et al., Concurrent validity of the Bayley scales of infant development II motor scale and the Peabody developmental motor scales-2 in children with developmental delays, Pediatr. Phys. Ther. 16 (2004)149-156.

[72] C. Missiuna, N. Pollock, Beyond the norms: need for multiple sources of data in the assessment of children, Phys. Occup. Ther. Pediatr. 15 (1995) 57-74.

[73] J. Williams, C. Hyde, A. Spittle, Developmental coordination disorder and cerebral palsy: is there a continuum? Current Developmental Disorders Reports 1 (2014) 118-124, https://doi.org/10.1007/s40474-014-0009-3.

\section{Further reading}

[74] J. Squires, D.D. Bricker, L. Potter, Ages and Stages Questionnaires User's Guide, Brookes Publishing Company, 1995.

[75] L. McCarron, McCarron Assessment of Neuromuscular Development: Fine and Gross Motor Abilities (Revised), McCarron-Dial Systems, 1997.

[76] S.L. Kemp, M. Korkman, in: Essentials of NEPSY Assessment, first ed., Wiley, Hoboken, 2001.

[77] J. Squires, D.D. Bricker, E. Twombly, in: Ages \& Stages Questionnaires, third ed., Paul H. Brookes, Baltimore, MD, USA, 2009 (ASQ-3).

[78] M.C. Piper, J.A.I.M.S. Darrah, Motor Assessment of the Developing Infants, W.B. Saunders, 1994.

[79] S.E. Henderson, D.A. Sugden, Movement Assessment Battery for Children, Psychological Corporation, London, 1992.

[80] W. Wu, C.F. Cheng, H.H. Lu, S.C. Chiu, Vineland Adaptive Behavior Scale Chinese Version, Psychological Publishing, 2004. 
[81] B.D. Bruininks, R.H. Bruininks, in: Bruininks-Oseretsky Test of Motor Proficiency, second ed., PsychCorp, Bloomington, 2010 (BOT-2).

[82] N.C. Bayley, Bayley Scales of Infant Development, Psychological Corporation, (NY, 1969.

[83] M.R. Folio, R.R. Fewell, Peabody Developmental Motor Scales and Activity Cards. Manual, DLM Teaching Resources, 1983.

[84] R.H. Bruininks, Bruininks-Oseretsky Test of Motor Proficiency, American Guidance Service Circle Pines, MN, 1978.

[85] S.M. Van der Meulen Bf, Bayley Developmental Scales Manual - Dutch Version (BOS2-30), Swets and Zeitlinger, Lisse, 1983.

[86] S.E. Henderson, D.A. Sugden, A.L. Barnett, B.C.M. Smits-Engelsman, Movement assessment battery for children, in: Dutch Version), second ed., Pearson Assessment and information B.V, 2010.

[87] D. McCarthy, McCarthy Scales of Children's Abilities, Psychological Corporation, 1972.

[88] K. Beery, Developmental Test of Visual Motor Integration, Follet, 1967.

[89] N. Bayley, B, in: Ayley Scales of Infant Development, second ed., The Psychological Corporation, 1993.

[90] B.R.S. Van der Meulen, H. Spelberg, M. Smrkovsky, in: Bayley Scales of Infant Development, second ed., Swets Test Publishers, 2002 (Dutch version).

[91] E. Hemgren, K. Persson, A model for combined assessment of motor performance and behaviour in 3-year-old children, Ups. J. Med. Sci. 104 (1999) 49-85, https://doi.org/10.3109/03009739909178955.

[92] D. Stott, F. Moyes, Manual: Test of Motor Impairment (Henderson Revision), Brook International, Guelph, Canada, 1984.

[93] B. Holle, K. Bönnelycke, E. Kemp, L. Mortensen, Motorisk-Perceptuell Utveckling, Psykologiförlaget AB, 1977.

[94] R. Griffiths, The Abilities of Young Children: A Comprehensive System of Mental Measurement for the First Eight Years of Life. (Griffiths Mental
Development Scales), The Test Agency, 1976.

[95] B.C.L. Touwen, Examination of the Child with Minor Neurological Dysfunction, William Heinemann, 1979.

[96] S.E. Henderson, D.A. Sudgen, A. Barnett, in: Movement Assessment Battery for Children, second ed., Harcourt Assessment, 2007.

[97] B. Smits-Engelsman, Dutch Manual Movement Assessment Battery for Children, Swets en Zeitlinger, Lisse, 1998.

[98] M. Korkman, U. Kirk, S. Kemp, NEPSY II: Clinical and Interpretive Manual, Psychological Corporation, 2007.

[99] R.-M.H. Schoemaker Mm, de Kloet AJ (original author Wilson BN) Developmental Coordination Questionnaire - Dutch Version, Sophia Revalidatie, 2007.

[100] M. Hadders-Algra, The Neurological Examination of the Child with Minor Neurological Dysfunction, Mac Keith Press, 2010.

[101] Beery The Beery-Buktencia, Developmental Test of Visual-Motor Integration. Administration, Scoring and Teaching Manual, fourth ed., Modern Curriculum Press, 1997.

[102] T.B. Brazelton, Neonatal Behavioral Assessment Scale, J.B. Lippincott Co., Philadelphia, 1984.

[103] Y.R. Burns, R.M. Ensbey, M.A. Norrie, The neuro-sensory motor developmental assessment Part 1: development and administration of the test, Aust. J. Physiother. 35 (1989) 141-149, https://doi.org/10.1016/S0004-9514(14) 60503-1.

[104] M.M. Palmer, K. Crawley, I.A. Blanco, Neonatal Oral-Motor Assessment scale: a reliability study, J. Perinatol.: official journal of the California Perinatal Association 13 (1993) 28-35.

[105] M. Folio, R. Fewel, in: Peabody Developmental Motor Scales: Examiner's Manual, 2nd. edition, Pro-ED Inc, 2000. 